\title{
On the Practical Implementation of VFDM-based Opportunistic Systems: Issues and Challenges
}

\author{
Marco Maso ${ }^{1,2}$, Leonardo S. Cardoso ${ }^{3}$, Ejder Bastug ${ }^{4}$, \\ Nguyen Linh-Trung ${ }^{5}$, Mérouane Debbah ${ }^{1}$, Ozgur Ozdemir ${ }^{4}$ \\ 1 Alcatel-Lucent Chair on Flexible Radio, SUPÉLEC, Gif-sur-Yvette, France \\ ${ }^{2}$ Department of Information Engineering, University of Padova, Italy \\ ${ }^{3}$ CITI Laboratory, INSA - Lyon, France \\ ${ }^{4}$ Department of Electrical and Electronics Engineering, Fatih University, Istanbul, Turkey \\ ${ }^{5}$ Fac. Electronics and Telecommunications, VNU University of Engineering and Technology, Vietnam
}

Correspondence: Marco Maso, marco.maso@supelec.fr

Manuscript communication: received 26 April 2012, accepted 9 May 2012

\begin{abstract}
Vandermonde-subspace frequency division multiplexing (VFDM) is a physical layer technique for cognitive twotiered networks, allowing for the coexistence of an orthogonal frequency division multiplexing (OFDM) legacy system and a cognitive secondary system in a time division duplex mode. It consists of a linear null-space precoder used by the secondary transmitter to effectively cancel the interference towards one or more primary receivers, while guaranteeing a non-negligible rate to a served secondary receiver. In this work, we propose an implementation of an experimental test-bed using the new SDR4All platform developed at the Alcatel-Lucent Chair on Flexible Radio (SUPELEC) to take a step towards a proof of concept of a VFDM-based system. We focus on the secondary link, where an opportunistic transmitter/receiver pair communicates over moderately frequency selective channels, characterized by very short root mean square (r.m.s.) delay spreads and non uniform power delay profiles (PDP). The obtained results show the practical feasibility of a VFDM transmission over a secondary link. However, a significant bit error rate (BER) loss with respect to the previously shown achievable theoretical performance is evident. A thorough analysis of the structure of the VFDM precoder is carried out and the impact of the channel characteristics on the performance of the opportunistic system is discussed. Numerical findings demonstrate that the potential BER drop can be addressed by designing a suitable flexible receiver able to deal with the effect induced by non uniform PDP and short r.m.s. delay spread channels.
\end{abstract}

Keywords- Cognitive overlay network, interference cancellation, small-cells, VFDM.

The paper was presented in part at IWCS 2011, Hanoi, Vietnam.

\section{INTRODUCTION}

In [1], we proposed a novel technique for an overlay cognitive network deployment called Vandermondesubspace frequency division multiplexing (VFDM). A secondary transmitter adopting VFDM can transmit over the same band as an orthogonal frequency division multiplexing (OFDM) primary system, while canceling its interference to the latter. Despite the absence of coordination between the two systems, a linear precoder that projects the transmitted signal onto the nullspace of the interfering link between the secondary transmitter and one or more primary receivers can always be devised [2]. This is accomplished by exploiting the redundancy added at the primary transmitter to reduce the inter-symbol interference (ISI) and interblock interference (IBI) which are caused by the multipath nature of the channel. In other words, VFDM exploits the frequency selectivity of Rayleigh fading multipath channels to identify transmit opportunities and devise a suitable precoder to null the cross-tier interference when perfect channel state information (CSI) is available at the secondary transmitter. Different from other interference management techniques for multicell networks [3]-[5], VFDM does not require either cooperation/coordination between the two considered cells, or multiple antenna installation at the transmitter/receiver.

In this contribution, we propose a first implementation of a cognitive VFDM transmitter/receiver pair prototype, based on the SDR4All platform [6]. This demonstrator focuses on the VFDM secondary link and serves as a further step towards an upcoming proof of concept of the full VFDM system. On the one hand, the outcome of our test demonstrates the feasibility of a VFDM-based secondary transmission. On the other hand, a significant BER detriment w.r.t. to the theoretical results provided in [1] is obtained. To investigate this, the statistical behavior of the channel is obtained by means of training symbols evaluation at the receiver. The estimated channel is shown to be characterized by a short root mean square (r.m.s.) delay spread and a fast decaying exponential power delay profile (PDP), thus very different from the Rayleigh 
fading channel model with uniform PDP considered in the analysis proposed in [1]. A thorough analysis is performed to better understand the structure of the null-space precoder for a short r.m.s. delay spread and a non-uniform PDP. By adopting a snapshot-based approach, we identify the relationship between the statistics of the channel and the power profile of the VFDM precoded signal. Our findings enlighten the importance of a suitable receiver architecture at the secondary receiver, that must be able to capture the effect induced by the channel statistics on the power profile of the transmitted/received signal. Numerical results reinforce this intuition and show a significant spectral efficiency enhancement, yielding results that approach the theoretical bounds and providing useful insights for future improvement of the VFDM test-bed.

This paper is organized as follows. In Section 2, we introduce the considered cognitive channel model. VFDM is described in Section 3. We describe the SDR4All platform in Section 4. In Section 5, we present the implementation guidelines for our prototype, transmitter/receiver side. In Section 6, we show some experimental results obtained after a set of transmissions. A thorough study of the precoder structure and power distribution are presented in Section 7 and 8 respectively. Finally, conclusions and future research directions are discussed in Section 9.

In this work, we adopt the mathematical notations as described in the following. A lower case italic symbol (e.g., b) denotes a scalar value, a lower case bold symbol (e.g., b) denotes a vector, an upper case bold symbol (e.g., B) denotes a matrix. $[\mathbf{B}]_{m n}$ denotes a matrix element at the row $m$ and the column $n$. An $\mathbf{I}_{\mathrm{N}}$ denotes the identity matrix of size $N$. The transpose conjugate operator on a matrix is denoted by the $\mathrm{H}$ superscript (e.g., $\mathbf{B}^{\mathrm{H}}$ ), whereas the Moore-Penrose inverse matrix is denoted by $+\left(\right.$ e.g., $\left.\mathbf{B}^{\dagger}\right)$. All vectors are columns, unless otherwise stated.

\section{SCENARIO}

Consider a transmission performed by two transmitter/receiver (TX/RX) pairs, denoted as primary (TX1/RX1) and secondary (TX2/RX2) system respectively. The latter wishes to communicate over the same band as the former, thus the resulting model is the cognitive interference channel (CIC), depicted in Figure 1. Wireless communications are typically affected by multi-path signal propagation, resulting in frequency selectivity of the channels. Recent standards (e.g., LTE [7]), propose block transmission systems to combat this phenomenon and provide high data rates. Then a natural choice is to assume that TX1 adopts a block transmission system, i.e., an $N$ subcarrier classical OFDM with cyclic prefix size of $L$. According to this scheme, once time and frequency synchronizations have been achieved, RX1 discards the $L$ leading symbols of each received OFDM symbol/block to eliminate ISI and IBI and equalize the signal using single-tap linear equalizers. Analogously, we assume that the same

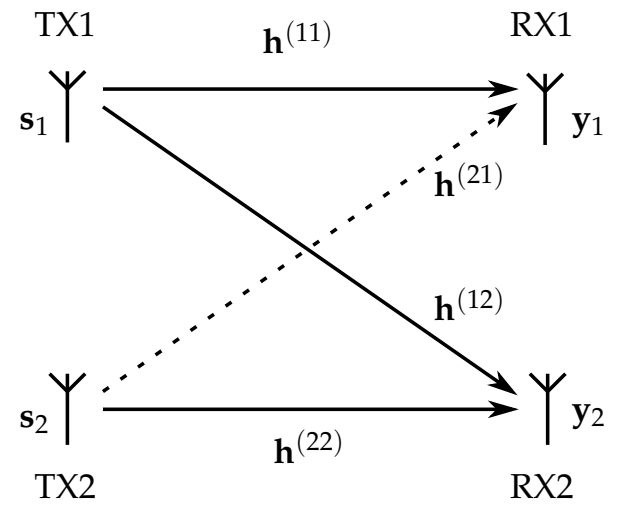

Figure 1. Cognitive interference channel model.

operations are performed at $\mathrm{RX} 2$ that consequently acts as an OFDM-like receiver. This choice is due both to the aforementioned physical argument (ISI and IBI suppression) and to architectural reasons enhancing the flexibility of the considered model, i.e., RX1 can act as $\mathrm{RX} 2$ and vice-versa with a simple software reconfiguration.

The primary system is the legal licensee of the spectrum and is oblivious of the existence of the secondary (or opportunistic) one. Therefore, no signal processing is implemented at the former to mitigate the cross-tier interference generated to the latter. Conversely the opportunistic system has to adhere to one of the interference management policies prescribed by the cognitive radio paradigm, i.e., cancelation (overlay or interweave approach) or mitigation (underlay approach) [8], to protect the licensee system from the undesired cross-tier interference. Typically, the opportunistic system exploits its cognitive capabilities to acquire side knowledge on the transmission of the primary system. Depending on the amount and nature of available information, the secondary transmit signal can be shaped to assume specific interference properties at the primary receiver.

In the considered scenario, the redundancy adopted at TX1 (i.e., cyclic prefix) to deal with the multipath propagation of the signal is not used to extract information at RX1. Therefore, TX2 can exploit these unused resources to design the opportunistic transmission such that the desired interference constraint is respected. We know from [1] that in such scenarios an overlay cognitive approach can be adopted, and a cross-interference cancelation linear precoder based technique called VFDM is implementable at TX2 if perfect CSI is available.

\section{Vandermonde-Subspace Frequency Division MultipLEXING}

Consider the modulated symbol vector at TX1. Let $\mathbf{s}_{1} \in \mathbb{C}^{N \times 1}$ be a comples zero mean unit norm input symbol vector. The OFDM transmit symbol vector $\mathbf{x}_{1} \in \mathbb{C}^{(N+L) \times 1}$ is then

$$
\mathbf{x}_{1}=\mathbf{A F}^{-1} \mathbf{s}_{1},
$$


where $\mathbf{A}$ is an $(N+L) \times N$ cyclic prefix insertion matrix and $\mathbf{F} \in \mathcal{C}^{N \times N}$ a unitary discrete Fourier transform $(\mathrm{DFT})$ matrix with $[\mathbf{F}]_{(k+1)(l+1)}=\frac{1}{\sqrt{N}} e^{-i 2 \pi \frac{k l}{N}}$ for $k, l=$ $0, \ldots, N-1$.

Similarly, we let $\mathbf{s}_{2} \in \mathbb{C}^{L \times 1}$ be the zero mean unit norm input symbol vector at TX2 [1] then the coded transmit symbol at TX2 can be written as

$$
\mathbf{x}_{2}=\mathbf{E s}_{2},
$$

where $\mathbf{E} \in \mathbb{C}^{(N+L) \times L}$ is the linear VFDM precoder [1]. Let $\mathbf{h}^{(i j)} \sim \mathbb{C} \mathcal{N}\left(0, \mathbf{I}_{l+1} /(l+1)\right)$ be i.i.d. Rayleigh fading channel vectors of $l+1$ taps, and we define $\mathcal{T}\left(\mathbf{h}^{(i j)}\right) \in$ $\mathbb{C}^{N \times(N+L)}$ as a Toeplitz matrix representing the convolution of the transmit symbol vectors with the channel. Consequently, the received signals at $\mathrm{RX} 1$ and $\mathrm{RX} 2$ are respectively

$$
\begin{aligned}
& \mathbf{y}_{1}=\mathbf{F}\left(\mathcal{T}\left(\mathbf{h}^{(11)}\right) \mathbf{x}_{1}+\mathcal{T}\left(\mathbf{h}^{(21)}\right) \mathbf{x}_{2}+\mathbf{n}_{1}\right) \\
& \mathbf{y}_{2}=\mathbf{F}\left(\mathcal{T}\left(\mathbf{h}^{(22)}\right) \mathbf{x}_{2}+\mathcal{T}\left(\mathbf{h}^{(12)}\right) \mathbf{x}_{1}+\mathbf{n}_{2}\right)
\end{aligned}
$$

where $\mathbf{n}_{1}$ and $\mathbf{n}_{2}$ are the additive white Gaussian noise (AWGN) vectors of length NN. Perfect time and frequency synchronizations at the receiver in both systems are assumed. In the considered overlay scenario, TX2 must process its signal such that RX1 does not see any residual interference after the cyclic prefix removal, regardless of the distribution or the realization of $\mathbf{s}_{2}$. This is in contrast with alternative approaches for cognitive network deployment that limit the maximum power used by the secondary system [9], hence limiting its usefulness mainly to short range communications [10]. By looking at (3), this implies that $\mathcal{T}\left(\mathbf{h}^{(21)}\right) \mathbf{x}_{2}=$ $\mathcal{T}\left(\mathbf{h}^{(21)}\right) \mathbf{E s}_{2}=\mathbf{B 0}$, for all $\mathbf{s}_{2} \in \mathbb{C}^{L \times 1}$.

Such a linear precoder projects the transmitted signal onto the null-space of the interfering channel from TX2 to RX1, thus completely cancels the interference if perfect CSI at the secondary transmitter (CSIT) is available. In [11], we showed that $\mathbf{E}$ can be computed as a Vandermonde matrix which is defined in [12]. Alternatively, for ill-conditioned $\mathcal{T}\left(\mathbf{h}^{(21)}\right)$, a Gram-Schmidt orthonormalization of the original Vandermonde matrix or a singular value decomposition (SVD) based approach may be preferable [1], [13].

\section{SDR4ALL}

In this work, the VFDM test-bed is implemented on the SDR4All platform, a novel hardware/software solution developed for teaching and development purposes in telecommunications and software radio [6]. The USB plug and play hardware part (i.e., USRP) including filters, amplifiers and oscillators, is responsible for the communication over the USB link, analogto-digital/digital-to-analog conversions and sampling. The RF circuitry, responsible of the analog signal generation, operates in the widely popular $2.4 \mathrm{GHz}$ ISM band, chosen by standards such as $802.11(\mathrm{~b} / \mathrm{g})$, Bluetooth and WiMAX. Furthermore, standard isotropic antennas made for the 2.4 and $2.49 \mathrm{GHz}$ ISM band are adopted. The main parameters of the SDR4All hardware are provided in Table I. The baseband processing
Table I

PARAMETERS FOR THE HARDWARE PART.

\begin{tabular}{c|c}
\hline parameter & value \\
\hline Operating band & ISM $2.4 \sim 2.49 \mathrm{GHz}$ \\
Baseband filtering & $20 \mathrm{MHz}$ \\
Channels & 1 to $13(802.11)$ \\
Total TX power & up to $50 \mathrm{~mW}$ \\
Data bandwidth & up to $16 \mathrm{MHz}$ \\
\hline
\end{tabular}

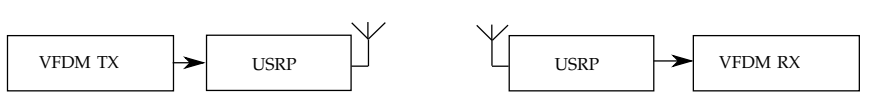

Figure 2. Experimental setup.

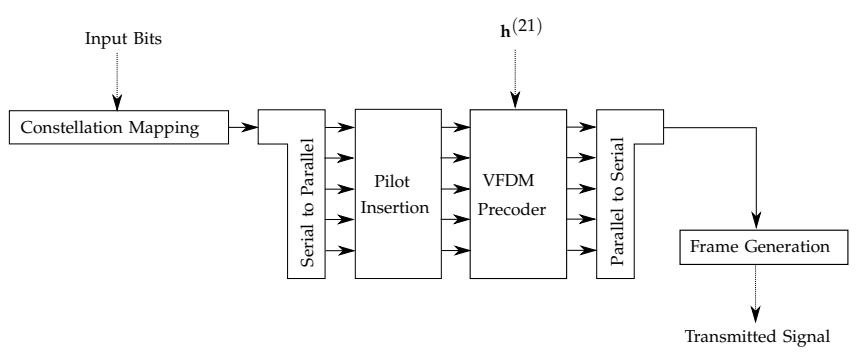

Figure 3. VFDM TX block diagram.

is provided by a user-friendly software toolbox, developed specifically to this end [14], operating in Matlab environment and providing full communication chains as well as basic communication blocks. A dedicate non real-time driver was built to allow the toolbox to communicate with a single daughter-board inside the USRPs. Nevertheless, multi-board, real-time operations will be supported in future firmware releases.

\section{VFDM IMPLEMENTATION}

The block scheme of the implemented experimental setup is depicted in Figure 2. As previously stated, in this contribution we focus on the secondary link, thus we consider the data transmission from TX2 to RX2. Consequently, no interference channel estimation is performed by TX2. We assume that the null-space precoder is derived using an $L+1$ path Rayleigh fading channel realization $\mathbf{h}^{(21)}$, generated for test purposes and used by the VFDM block as hypothetical interference channel between TX2 and RX1. We first analyze the secondary transmitter. The kernel driver of the toolbox handles the data streams and communicates with the USRP over the USB link. Further details about the configuration and logic of the USRP's hardware can be found in [15].

\subsection{VFDM Baseband Transmitter}

The block diagram of the VFDM transmitter is shown in Figure 3. The input bits are mapped into $M L$ symbols that are successively parallelized into $L$ streams, according to the requirements of the VFDM precoder as illustrated in Section 3. The resulting stream matrix $\mathbf{S} \in \mathbb{C}^{L \times M}$ is fed to the the pilot insertion block, where the transmit frame is built by alternating groups of symbol blocks and pilot blocks. Let $N_{\text {Pilots }}$ be a parameter defining the number of groups of pilot blocks inside 


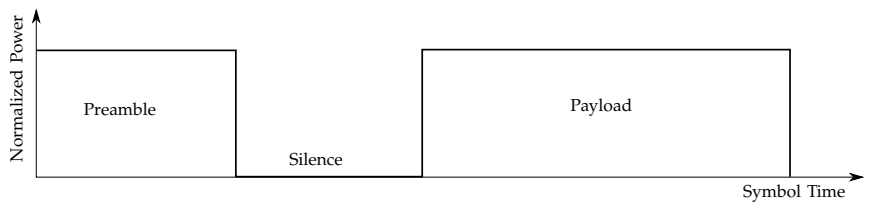

Figure 4. Frame Structure.

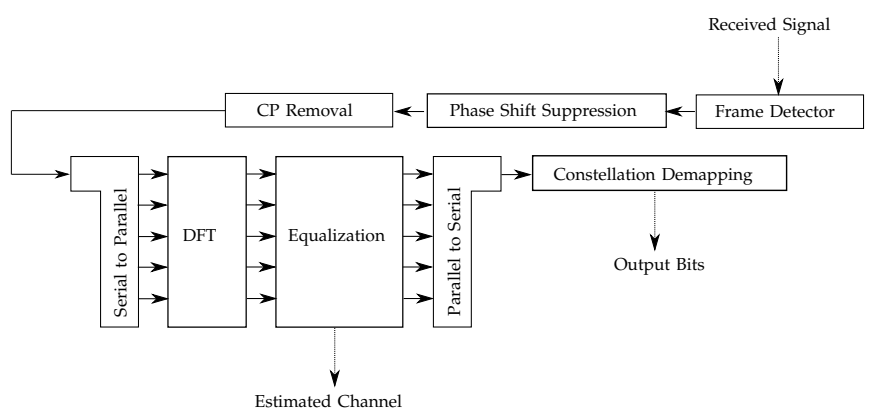

Figure 5. VFDM RX block diagram.

one frame. The pilot insertion procedure is articulated in three steps:

1) The input data stream is divided into $N_{\text {Pilots }}$ groups of blocks denoted as $\mathbf{D}_{j} \in \mathbb{C}^{L \times\left(M / N_{\text {Pilots }}\right)}$, with $j=1, \ldots, N_{\text {Pilots. }}$.

2) A pilot symbol matrix $\mathbf{P}$ of size $L \times M_{\text {Pilots }}$ is generated, such that

$$
\left\{\begin{array}{l}
{[\mathbf{P}]_{(k+1)(l+1)} \alpha e^{-i 2 \pi \frac{k l}{M_{\text {Pilots }}},}} \\
k=0, \ldots, L-1 \\
l=0, \ldots, M_{\text {Pilots }}
\end{array}\right.
$$

3) The uncoded payload $\mathbf{T} \in \mathbb{C}^{L \times\left(M+N_{\text {Pilots }} M_{\text {Pilots }}\right)}$ is composed by alternating $\mathbf{P}$ to $\mathbf{D}_{j}$, as follows

$$
\mathbf{T}=\left[\mathbf{D}_{1}\left|\mathbf{P}_{1}\right| \mathbf{D}_{2}\left|\mathbf{P}_{2}\right| \mathbf{D}_{3} \mid \cdots\right],
$$

where $\mathbf{P}_{j}$, with $j=1, \ldots, N_{\text {Pilots, }}$ is the $j^{\text {th }}$ pilot symbol matrix repetition.

At this stage, the VFDM precoder block generates the coded payload of the transmission, successively serialized to be ready for the frame generation. Let $b, c \in \mathbb{N}_{\star}{ }^{1}$ be scaling parameters. The frame generator adds to the payload a preamble known at the receiver, characterized by the following structure:

1) A Golay complementary sequence $\mathbf{g}$ [16] of length $N$, taking values in $\{1+i,-1-i\}$, for time/frequency synchronization purposes at the receiver.

2) A constant sequence of symbols o $\in\{1+i\}^{N}$, introduced to assure correct detection of phase offset variations.

3) A guard time of size $c N$. The final frame structure is depicted in Figure 4.

\subsection{VFDM Baseband Receiver}

The block diagram of the VFDM receiver is represented in Figure 5. The first operation performed at the receiver is the frame detection. This is accomplished by means of suitable time and symbol level

\footnotetext{
${ }^{1}$ To avoid ambiguity in the preamble definition, we consider $\mathbb{N}_{\star}$ as the set of natural number excluding 0 .
}

synchronization, exploiting the structure of the received frame. In the proposed implementation, $\mathrm{RX} 2$ exploits the known payload structure to identify the starting point of the VFDM frame with accuracy. As a first step, the cross-correlation between the received signal $\mathbf{y}$, and the known Golay sequence $\mathbf{g}$ is computed as

$$
R(n) \triangleq \sum_{m} \mathbf{y}^{*}(n) \mathbf{g}(n+m) \text {. }
$$

The Golay complementary sequence is characterized by good autocorrelation properties, presenting a clear peak for $m=0$. Therefore, $\mathrm{RX} 2$ can detect $\hat{n}$, which is the estimated starting point of the frame, by taking

$$
\hat{n}=\max _{n} R(n)
$$

In any communication systems, imperfections in the phase lock loop (responsible for generating the carrier frequency at the chosen central frequency $f_{\mathrm{c}}$ ), channel rotations and thermal noise can induce a phase shift of the received frame at the receiver. The accuracy of the decoding can be severely affected by an unsuppressed phase shift. Therefore, a two-step procedure is adopted in our test-bed to address this issue. Despite the existence of other more refined techniques in the literature, the following solution provides simplicity and low complexity operations, and it is adopted by many existing standards [17], [18]. By construction, inside the preamble, the sequence $\mathbf{o}$ is composed of symbols having the same phase. Therefore, if we denote $\phi|\cdot|$ as the phase of a given complex value, RX2 can obtain a first coarse phase shift estimation as

$$
\hat{\phi}_{\mathrm{c}}=\frac{1}{b N} \sum_{m=\hat{n}}^{\hat{n}+N(b+1)-1}\left(\phi\left|r_{m+1}\right|-\phi\left|r_{m}\right|\right),
$$

where each subsequent phase offset computation is averaged to compensate for phase noise. At this stage, a first compensation takes place. Afterwards, $\hat{\phi}_{\mathrm{f}}$, hereafter fine phase shift, is estimated. Similar to what is described in [17], $\mathbf{o}$ is divided in two equal portions, $\mathbf{o}_{1}$ and $\mathbf{o}_{2}$, and $\mathrm{RX} 2$ can estimate $\hat{\phi}_{\mathrm{f}}$ as

$$
\hat{\phi}_{\mathrm{f}}=\frac{4}{(b N)^{2}} \sum_{m=\hat{n}}^{\hat{n}+\frac{N(b+1)}{2}-1} \phi\left|r_{m} r_{\frac{N(b+1)}{2}}^{*}\right| .
$$

The fine phase shift compensation ends the preamble processing. After a cyclic prefix removal operation, the stream is parallelized and a DFT is performed, according to the model introduced in Section 3. At this stage, each symbol block has size $N$, number of carriers used in the OFDM primary system. If we let $\tilde{\mathbf{P}}_{i}$ be the $i^{\text {th }}$ repetition of $\mathbf{P}$ corrupted by the channel and $\tilde{\mathbf{D}}_{i}$ be the $i^{t h}$ received data matrix of size $N \times \frac{M}{N_{\text {Pilots }}}$, we can write the frequency domain representation of the received frame as

$$
\tilde{\mathbf{T}}=\left[\tilde{\mathbf{D}}_{1}\left|\tilde{\mathbf{P}}_{1}\right| \tilde{\mathbf{D}}_{2}\left|\tilde{\mathbf{P}}_{2}\right| \tilde{\mathbf{D}}_{3} \mid \cdots\right] \in \mathbb{C}^{N \times\left(M+N_{\text {Pilots }} M_{\text {Pilots }}\right)} .
$$

Note that, the equalizer can benefit from the repetition of the matrix $\mathbf{P}$ inside the frame to update the channel estimation frequently. Each data block is equalized using the channel estimation provided by the previous 
$\mathbf{P}$ evaluation. Consequently, in general, RX2 does not need any information about the coherence time of the channel a priori. Furthermore, a frequent channel estimation can mitigate the impact of an overly noisy environment and improve the overall decoding performance. By definition, $\mathbf{P}_{i} \mathbf{P}_{i}^{\mathrm{H}}=\mathbf{I}_{L}$, thus the $i^{\text {th }}$ equivalent channel estimation is obtained by pilot evaluation as follows:

$$
\widehat{\mathbf{H}}_{i}=\tilde{\mathbf{P}}_{i} \mathbf{P}^{\mathrm{H}} .
$$

Finally, a simple zero forcing equalizer [19] is implemented. The equalized payload can be written as $\widehat{\mathbf{T}}=\left[\widehat{\mathbf{D}}_{1}\left|\widehat{\mathbf{D}}_{2}\right| \ldots \mid \widehat{\mathbf{D}}_{\frac{M}{N_{\text {Pilots }}}}\right] \in \mathbb{C}^{N \times M}$, where

$$
\widehat{\mathbf{D}}_{i}=\mathbf{H}_{i}^{\dagger} \tilde{\mathbf{D}}_{i} .
$$

The received symbols are then serialized and demapped to obtain the output bit sequence.

\section{ExPERIMENTAl Results}

A test-bed composed of a TX/RX pair, managed by two laptops, as illustrated in Figure 6, has been set up to validate the proposed solution. As described in Section 4, the baseband signal processing is implemented at software level, exploiting a MATLAB $\mathbb{R}$ toolbox developed specifically to this end [14]. The SDR4All platform drives the hardware at both side of the transmission. In the considered scenario, TX2 does not send any trigger message to the receiver before starting the VFDM transmission. To make sure that $\mathrm{RX} 2$ is able to receive and buffer enough meaningful data, a repetition of the VFDM frame is transmitted. We note that the size of the transmit window can be set at software level using the SDR4All toolbox. TX2 performs a set of 1000 transmissions, such that statistically relevant results can be obtained. The main parameters used for system configuration are provided in Table II.

The frame structure discussed in Section 5.1, represented in Figure 4, contemplates a guard band (i.e., zero sequence) insertion between the preamble and the frame, used to compute a first estimate of the experienced signal-to-noise ratio (SNR) at the receiver. In fact, the receiver can evaluate the received power during the second part of the preamble (i.e., the constant sequence), and the noise power during the silence and compute their ratio. The power profile of the received frame at RX2 is shown in Figure 7. In this case, the

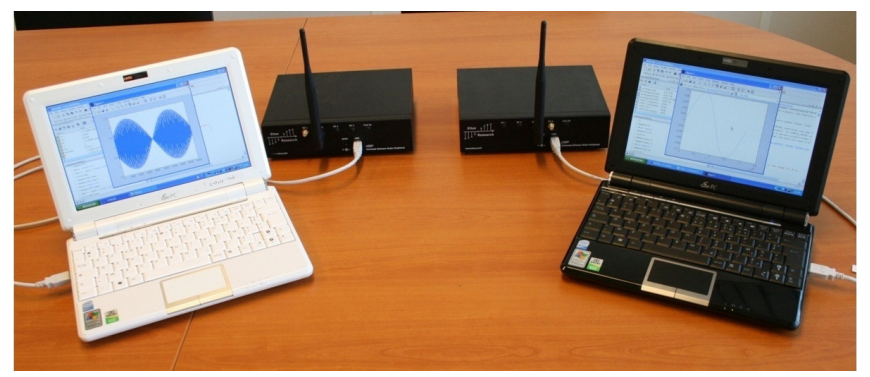

Figure 6. Transmission test-bed.
Table II

USER DEFINED PARAMETERS.

\begin{tabular}{c|c}
\hline parameter & value \\
\hline Carrier frequency & ISM $2.412 \mathrm{GHz}$ \\
Bandwidth & $4 \mathrm{MHz}$ \\
$N$ & 64 \\
$L$ & 8 \\
$N_{\text {Pilots }}$ & $M / 120$ \\
$M_{\text {Pilots }}$ & 10 \\
Golay sequence length & 64 \\
Constant sequence length & 2048 \\
Zeros sequence length & 128 \\
Modulation order & $4-\mathrm{QAM}$ \\
\hline
\end{tabular}

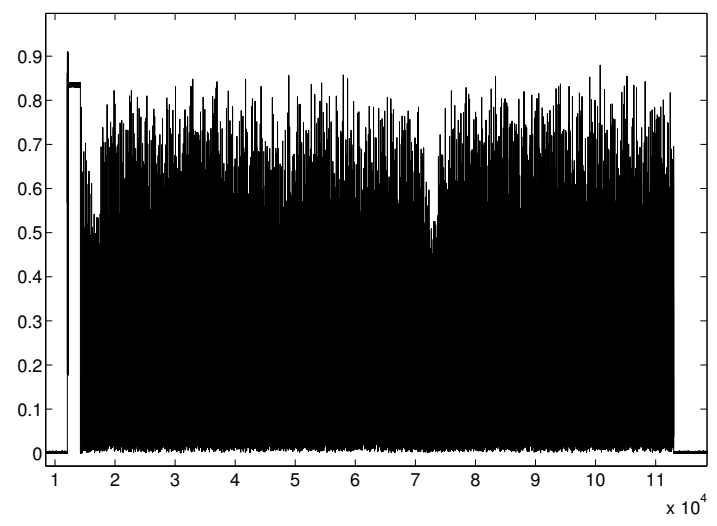

Figure 7. Receive frame at RX2.

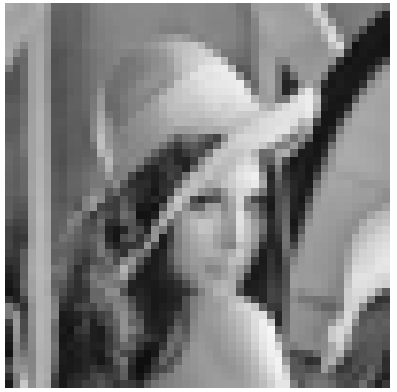

(a) Transmitted data

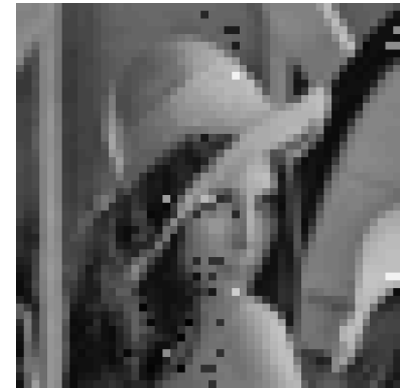

(b) Received data
Figure 8. Transmitted and received data.

preamble SNR is $25.1 \mathrm{~dB}$. We note that the payload exhibits a very irregular power profile, especially if compared to the preamble. This behavior is typically due to the peak-to-average power ratio (PAPR) problem. In particular, this implies that the payload SNR may be substantially different from the first estimation provided above. For the sake of simplicity, we focus on one of the aforementioned transmissions, whose outcome is presented in Figures 8(a) and 8(b). As a first observation, we note that the proposed scheme for a standalone VFDM transmission is correctly working. However, the number of faulty pixels in Figure 8(b) is unlikely result of a transmission performed at high SNR, whose bit error rate (BER) should be lower.

By computing the cumulative distribution function (CDF) of the BER, we characterize its behavior. The result of this operation is presented in Figure 9. By comparing these results to what we presented in [1], we see that the average BER in that contribution for 


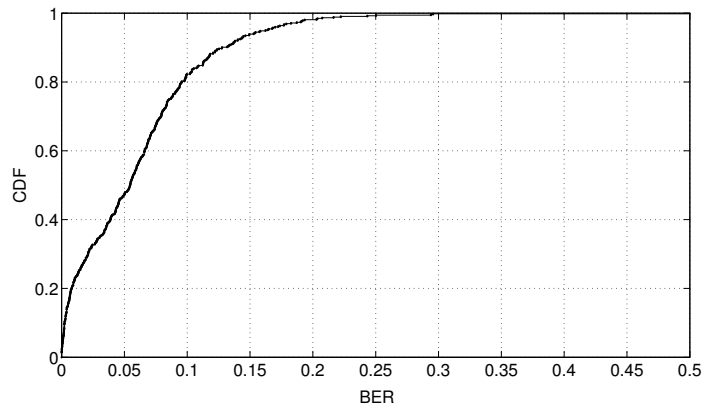

Figure 9. CDF of the BER.

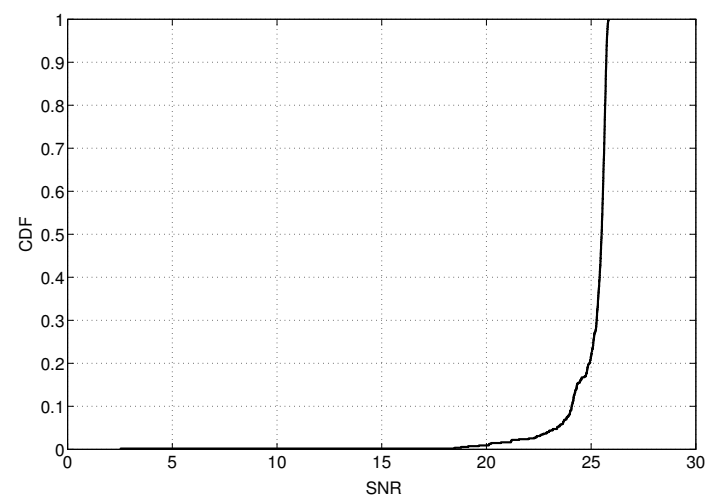

Figure 10. CDF of the SNR.

$\mathrm{SNR}=25 \mathrm{~dB}$ falls into the $10^{\text {th }}$ percentile of the BER of the current test. Interestingly, the computed average BER is 0.0603 that, according to [1], is the average BER of a transmission performed at SNR $\sim 9 \mathrm{~dB}$.

These findings demonstrate that the preamble SNR and the BER of the transmission are not in a direct relationship. Therefore, the experienced SNR for preamble and payload have likely very different values. This is confirmed by Figure 10, where the CDF of the preamble SNR is presented. The CDF shows an evident behavior, with an average value of SNR $\sim 25 \mathrm{~dB}$, confirming our previous results and pointing out the unsuitability of the preamble SNR measurement to give a correct estimate of the payload SNR. Accordingly, the behavior of the power profile of the payload has to be studied in order to fully characterize our system and address this issue.

\section{Precoder Analysis}

The experimental results obtained so far show that a secondary VFDM based transmission can experience significant SNR drops at the receiver, hence rate loss. In this section, we present an analysis of the VFDM precoder, in the presence of channels characterized by different PDPs and r.m.s. delay spreads, to investigate their impact on the precoder structure. We specifically target our efforts on TX2's transmission, thus we simplify the model presented in Section 3 and consider only the received signal component coming from the secondary system

$$
\begin{aligned}
& \mathbf{y}_{1}=\mathcal{T}\left(\mathbf{h}^{(21)}\right) \mathbf{E x}_{2} \\
& \mathbf{y}_{2}=\mathcal{T}\left(\mathbf{h}^{(22)}\right) \mathbf{E x}_{2}
\end{aligned},
$$

where the thermal noise has been ignored for simplicity. We recall that the parameters $N, L$ and $l$ define respectively the number of carriers, the cyclic prefix size and the number of paths of the channel, line-of-sight (LOS) component excluded. For the sake of simplicity we will consider that the channels in primary and secondary system are characterized by the same parameter $l$. Our goal is to understand how the precoder modifies the power profile of the input symbol vector $\mathbf{x}_{2}$ of size $L$.

We start by characterizing the structure of the precoder, by explicitly computing the solution of the linear system arising from the interference nulling constraint. In general, in practical OFDM implementations, the cyclic prefix is over dimensioned with respect to the number of channel paths, to avoid ISI and IBI. Therefore, we can write the considered channel matrices in the following equivalent form:

$$
\begin{aligned}
\mathcal{T}\left(\mathbf{h}^{(21)}\right) & =\left[\mathbf{0}_{N \times(L-l)} \mid \mathbf{H}_{\mathrm{p}}\right] \\
\mathcal{T}\left(\mathbf{h}^{(22)}\right) & =\left[\mathbf{0}_{N \times(L-l)} \mid \mathbf{H}_{\mathrm{s}}\right]
\end{aligned}
$$

For the sake of simplicity in the notation, we rewrite $\mathbf{h}^{(21)}=\left[h_{\mathrm{p} 1}, \ldots, h_{\mathrm{p}(l+1)}\right]$, and $\mathbf{h}^{(22)}=\left[h_{\mathrm{s} 1}, \ldots, h_{\mathrm{s}(l+1)}\right]$. We note that, $\mathcal{T}\left(\mathbf{h}^{(21)}\right)$ and $\mathcal{T}\left(\mathbf{h}^{(22)}\right)$ can assume different structures depending on the relationship between $L$ and $l$.

The secondary transmitter first derives a suitable null-space precoder $\mathbf{V}$ by solving the equation $\mathcal{T}\left(\mathbf{h}^{(21)}\right) \mathbf{V}=\mathbf{0}$, an orthonormalization of the columns of $\mathbf{V}$ is then computed to yield $\mathbf{E}$. The solution $\mathbf{V}=\left[\mathbf{v}_{1}^{\mathrm{T}}\left|\mathbf{v}_{2}^{\mathrm{T}}\right| \ldots \mid \mathbf{v}_{L}^{\mathrm{T}}\right]$ has the form

$$
\mathbf{V}=\left[\mathbf{r}_{1}^{T} \cdots \mathbf{r}_{L-l}^{T} \mid \mathbf{B}^{T}\right]^{T},
$$

with $\left\{\mathbf{r}_{1}, \ldots, \mathbf{r}_{L-l}\right\} \in \mathbb{C}^{1 \times L}$ vectors with random entries, and $\mathbf{B} \in \mathbb{C}^{(N+l) \times L}$ matrix such that $\mathbf{H}_{\mathrm{p}} \mathbf{B}=\mathbf{0}$. Consequently, the following sets of equations need to solved to find $\mathbf{B}$

$$
\begin{cases}b_{1 j} h_{\mathrm{p}(l+1)}+\ldots+b_{(l+1) j} h_{\mathrm{p} 1} & =0 \\ b_{2 j} h_{\mathrm{p}(l+1)}+\ldots+b_{(l+2) j} h_{\mathrm{p} 1} & =0 \\ \vdots & \vdots \\ b_{N j} h_{\mathrm{p}(l+1)}+\ldots+b_{(l+N) j} h_{\mathrm{p} 1} & =0 .\end{cases}
$$

By looking at (17), we can identify a system of $N$ equations in $N+l$ variables. Consequently, (17) is determined if and only if $l=0$, i.e., for LOS channels. Conversely, for $l>0$, i.e., for non LOS (NLOS) channels, the system is always under-determined no matter which $N, L$ and $l$ values are selected. Naturally this impacts significantly the structure of $\mathbf{B}$, hence we need to analyze the different cases separately.

\subsection{LOS Channels}

We start by considering LOS channels, i.e., $l=0$. As a consequence, $\mathbf{h}^{(21)}=\left[h_{\mathrm{p} 1}\right]$ and $\mathbf{h}^{(22)}=\left[h_{\mathrm{s} 1}\right]$, and we 
have

$$
\begin{aligned}
& \mathcal{T}\left(\mathbf{h}^{(21)}\right)=h_{\mathrm{p} 1}\left[\mathbf{0}_{N \times L} \mid \mathbf{I}_{N}\right] \\
& \mathcal{T}\left(\mathbf{h}^{(22)}\right)=h_{\mathrm{s} 1}\left[\mathbf{0}_{N \times L} \mid \mathbf{I}_{N}\right]
\end{aligned}
$$

where $h_{\mathrm{p} 1}, h_{\mathrm{s} 1} \in \mathbb{C}$ and $\mathbf{I}_{N}$ is an size- $N$ identiy matrix. By solving the linear system in (17), TX2 derives the trivial solution

$$
\mathbf{V}=\left[\begin{array}{c}
\mathbf{A}_{L \times L} \\
\mathbf{0}_{N \times L}
\end{array}\right],
$$

where $\mathbf{A}_{L \times L} \in \mathbb{C}^{L \times L}$ is a matrix with random entries. $\mathbf{E}$ is then trivially obtained as

$$
\mathbf{E}=\left[\begin{array}{c}
\mathbf{I}_{L \times L} \\
\mathbf{0}_{N \times L}
\end{array}\right] .
$$

Therefore, for LOS channels, the null-space precoder degenerates into a time division multiple access (TDMA) approach. TX2 transmits only over the first $L$ symbols of the OFDM block, and $\mathbf{B}=\mathbf{0}_{N \times L}$. The precoder in (19) implies also that $\mathbf{H}_{\mathrm{sS}} \mathbf{B}=\mathbf{B}$, and

$$
\mathcal{T}\left(\mathbf{h}^{(22)}\right) \mathbf{E}=\mathbf{0}_{(N+L) \times L}, \quad \forall h_{\mathrm{s} 1} \in \mathbf{C} .
$$

As a consequence, $\mathbf{y}_{2}=0$, and no symbol can be decoded. We argue that, for LOS channels, no linearly precoded transmission can be performed by a single antenna TX2 communicating with a secondary OFDM-like receiver, while guaranteeing the interference cancellation at the primary receiver. If the secondary transmitter removes the cyclic prefix, the SNR of the resulting $N$ symbols as well as the maximum secondary system achievable spectral efficiency is always equal to zero.

This result is not surprising in fact, if $l=0$, the $N$ variables in (17) do not have any available degree of freedom. By construction, they already appear in fully solvable form, as given by

$$
b_{i j} h_{\mathrm{p} 1}=0, \quad i=j,
$$

thus they are always identically equal to zero. Therefore, the considered homogeneous system can admit only the set of solutions in (19). This poses a potential harm to the effectiveness of the current scheme, and the only possible workaround is represented by a change in the signal model in the secondary system.

\subsection{NLOS Channels}

If $l>0$, then $\mathbf{h}^{(21)}=\left[h_{\mathrm{p} 1}, \ldots, h_{\mathrm{pl}}\right]$, and we can refer to the general model introduced in Section 3. In this case, the under-determined system of $N$ equations in $N+l$ variables in (17) does not present any variable in fully solvable form, and the set of the possible solutions highly hinges on the parameter $l$. In order to better characterize its impact on the precoder structure, we start our analysis from a simple case, $l=1$, that is a two-path channel.
7.2.1 Two-path channel: Consider a channel with two paths, including the LOS component, i.e., $l=1$. Therefore, $\mathbf{h}_{(21)}=\left[h_{\mathrm{p} 1}, h_{\mathrm{p} 2}\right]$ and $\mathbf{h}_{\mathrm{s}}=\left[h_{\mathrm{s} 1}, h_{\mathrm{s} 2}\right]$. In this case, we have

$$
\mathbf{H}_{\mathrm{p}}=\left[\begin{array}{ccccccc}
h_{\mathrm{p} 2} & h_{\mathrm{p} 1} & 0 & 0 & 0 & \ldots & 0 \\
0 & h_{\mathrm{p} 2} & h_{\mathrm{p} 1} & 0 & 0 & \ldots & 0 \\
\vdots & \ddots & & & & \ddots & \vdots \\
0 & 0 & 0 & \ldots & 0 & h_{\mathrm{p} 2} & h_{\mathrm{p} 1}
\end{array}\right]
$$

We know from [1] that $\operatorname{dim} \operatorname{ker} \mathcal{T}\left(\mathbf{h}^{(21)}\right)=L$, thus the number of columns of $\mathbf{V}$ is dependent on the cyclic prefix size. Consequently, in the following we will consider several values of $L$. Note that, $N=4$ if not differently stated.

For the simple case of $L=l=1$, a systematic recursive solution can be found

$$
b_{i j}=(-1)^{N+i} b_{N j}\left(\frac{h_{\mathrm{p} 1}}{h_{\mathrm{p} 2}}\right)^{N-i}
$$

where $b_{N j}$ has been arbitrarily chosen among all the variables, without loss of generality. In fact, in this case, any chosen variable could be factorized out and the behavior will always be depending on the ratio $h_{\mathrm{p} 1} / h_{\mathrm{p} 2}$. Therefore, when $L=l=1$, the precoder $\mathbf{V}$ degenerates into a column vector that, given the selected variable and $N=4$, can be written as

$$
\mathbf{V}_{[1 j]}=b_{4 j}\left[-\left(\frac{h_{\mathrm{p} 1}}{h_{\mathrm{p} 2}}\right)^{3},\left(\frac{h_{\mathrm{p} 1}}{h_{\mathrm{p} 2}}\right)^{2},-\frac{h_{\mathrm{p} 1}}{h_{\mathrm{p} 2}}, 1,-\frac{h_{\mathrm{p} 2}}{h_{\mathrm{p} 1}}\right]^{\mathrm{T}},
$$

and its orthonormalized version $\mathbf{E}_{[1 j]}=\frac{1}{\sqrt{D_{1}}} \mathbf{V}_{[1 j]}$, where

$$
D_{1}=\left|b_{4 j}\right|^{2}\left[\left|\frac{h_{\mathrm{p} 1}^{3}}{h_{\mathrm{p} 2}^{3}}\right|^{2}+\left|\frac{h_{\mathrm{p} 1}^{2}}{h_{\mathrm{p} 2}^{2}}\right|^{2}+\left|\frac{h_{\mathrm{p} 1}}{h_{\mathrm{p} 2}}\right|^{2}+\left|\frac{h_{\mathrm{p} 2}}{h_{\mathrm{p} 1}}\right|^{2}+1\right] .
$$

Unfortunately, this simple solution can not be extended to larger values of $L$ and $l$. In fact, when these two parameters are greater than 1 , the resulting equations yield very long and complex results thus, for the sake of readability, we present the corresponding analysis in Appendix. Nevertheless, the obtained results show that this approach leads to unmanageable computations. Despite the attempt to simplify the equations in order to identify repeating patterns, a systematic closed-form representation of $\operatorname{ker} \mathcal{T}\left(\mathbf{h}^{(21)}\right)$ depending on the three main parameters $(N, L$, and $l)$ is not derivable. Consequently, we have to face the analysis of the power distribution of the precoded symbols numerically.

\section{Power Distribution}

As seen in Appendix A, the complexity of the explicit precoder computation brings long and non trivial equations even for very simple configurations. This prevents us to characterize the power profile of the precoded signal systematically, even though we can 


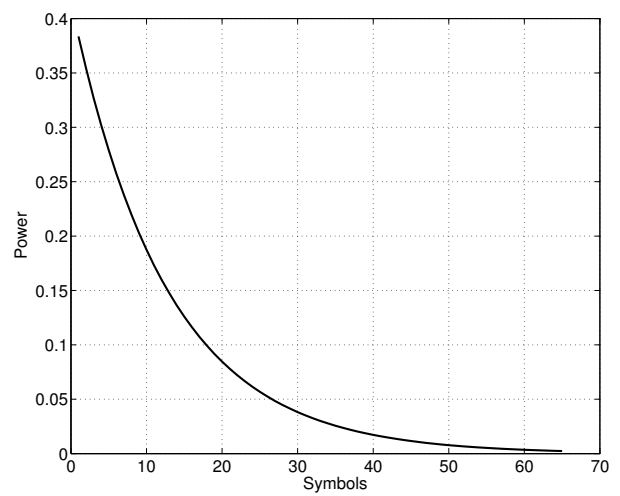

(a) $\left|\frac{h_{\mathrm{p} 1}}{h_{\mathrm{p} 2}}\right|<1$

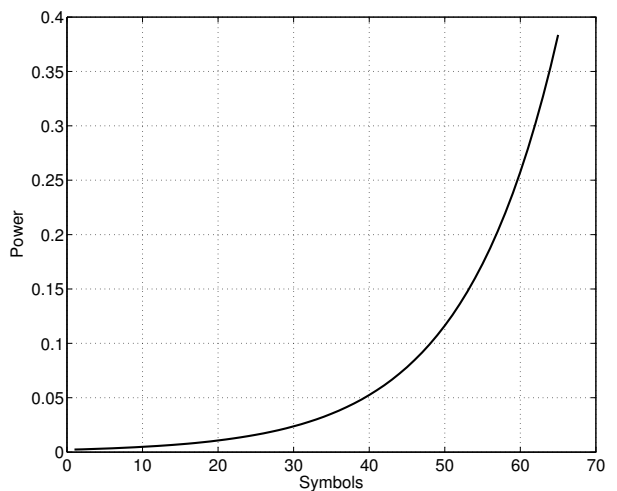

(b) $\left|\frac{h_{\mathrm{p} 1}}{h_{\mathrm{p} 2}}\right|>1$

Figure 11. Power profile of the column precoder when $L=l=1$.

infer useful insights and have a better understanding on how the power is distributed among the symbols. As a simple example, we can consider the case presented in Section 7.2 and analyze the power distribution along the vector given by (23), i.e., $L=l=1$. As can be inferred by looking at the equation, the ratio $\left|h_{\mathrm{p} 1} / h_{\mathrm{p} 2}\right|$ imposes an exponentially decreasing/increasing power profile to the precoded symbols. The corresponding outcome is depicted in Figures 11(a) and 11(b), where the trivial case $\left|h_{\mathrm{p} 1} / h_{\mathrm{p} 2}\right|=1$ is omitted. For practical purposes, the case $\left|h_{\mathrm{p} 1} / h_{\mathrm{p} 2}\right|>1$ is unlikely to be occurring in a real transmission, thus we focus on the scenario $\left|h_{\mathrm{p} 1} / h_{\mathrm{p} 2}\right|<1$. We can immediately notice from Figure 11(a) that the power is not uniformly distributed but, on the contrary, is concentrated during the first part of the symbols. Here the structured nature of $\operatorname{ker} \mathcal{T}\left(\mathbf{h}^{(21)}\right)$ assumes a precise characterization, regardless of the instances of the channel, whenever $\left|h_{\mathrm{p} 1} / h_{\mathrm{p} 2}\right|<1$ the power profile of the precoder has an exponential decay along the symbols. Moreover, the smaller $\left|h_{\mathrm{p} 1} / h_{\mathrm{p} 2}\right|$ the faster the decay.

If we move from the simple example to the more complicate cases in Section 7.2, we can see that, even if we can not easily deduce any characteristic of the power distribution along the precoded symbols, the PDP of the channel plays a fundamental role in this matter. In the following section, we will consider the same setup as in Section 3, and we will characterize numerically

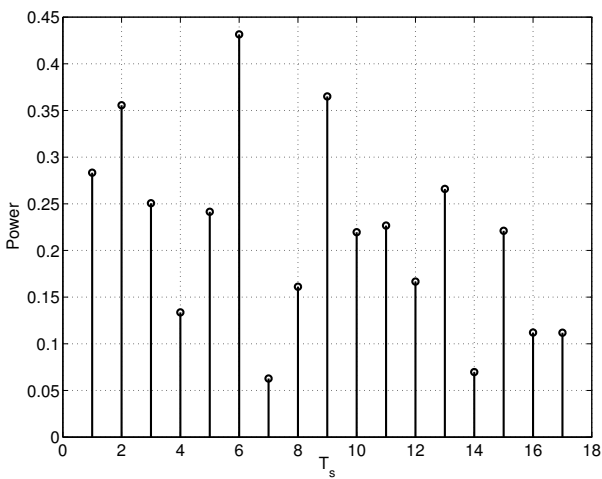

(a) Uniform

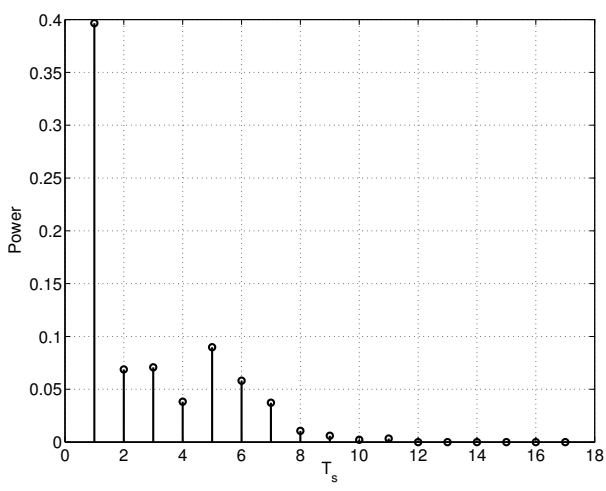

(b) Exponential, slow decay, $\frac{T_{s}}{\tau}=0.75$

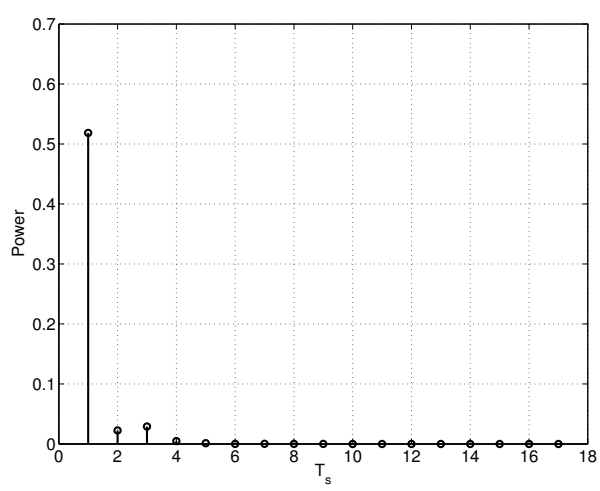

(c) Exponential, fast decay, $\frac{T_{s}}{\tau}=2.5$

Figure 12. PDP of the interference link.

the relationship between the PDP of the channel and the power distribution of the precoded symbols.

\subsection{PDP Importance}

In the following Monte-Carlo simulations, we assume that the OFDM reference system transmits over $N=$ 64 subcarriers, with a cyclic prefix size of $L=16$. Three different PDP models for the considered Rayleigh fading channel $\mathbf{h}_{\mathrm{p}}$ are considered, namely uniform, exponential with fast $\left(\frac{T_{s}}{\tau}=2.5\right)$ and slow $\left(\frac{T_{s}}{\tau}=0.75\right)$ decay, where $T_{S}$ is the sample time and $\tau$ is the r.m.s. delay spread. In Figure 12, three channel snapshots are depicted, one for each of the aforementioned PDP, where we represent the power associated to each path. 


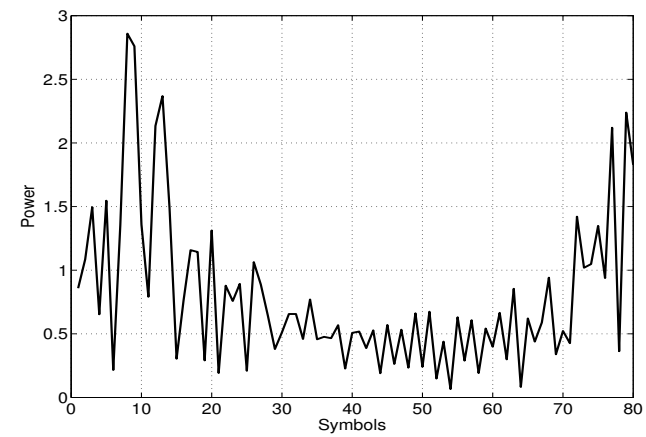

(a) Uniform PDP

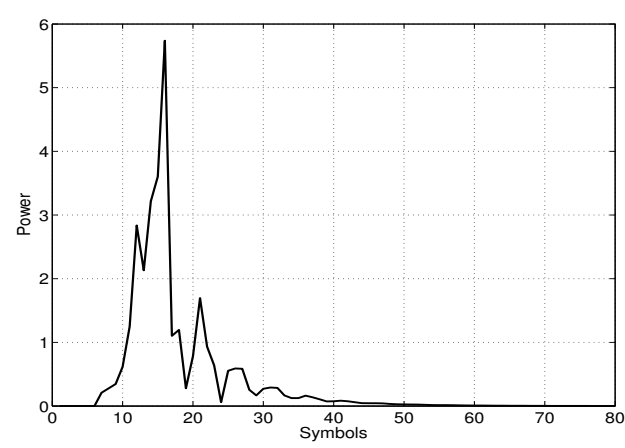

(b) Exponential PDP, slow decay, $\frac{T_{s}}{\tau}=0.75$

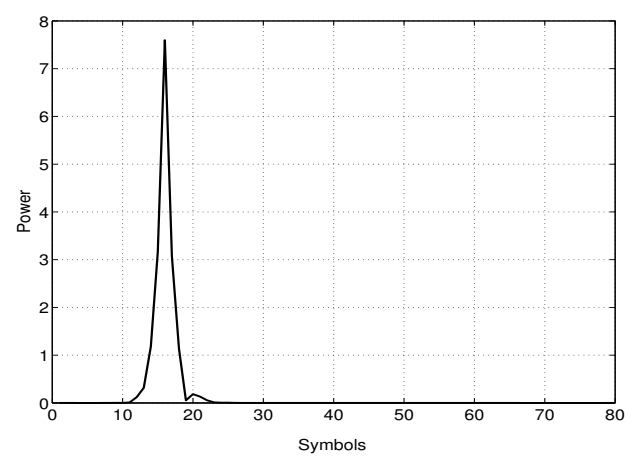

(c) Exponential PDP, fast decay, $\frac{T_{s}}{\tau}=2.5$

Figure 13. Power distribution of the precoded symbols.

We focus on these snapshots to understand how the power associated to the channel taps can induce a non uniform power distribution of the precoded VFDM symbols, shown in Figure 13. Even though not generalized, this approach is meant to show how channels presenting a given statistical description can influence the performance of the secondary system, as seen in the previous sections. Aside from the high PAPR that characterizes all the three samples, the impact of the delay spread and PDP of the channel on the power profile of the precoded transmit symbols is significant. The power is distributed over the whole duration of the symbol, even though not uniformly, only for uniform PDP, as depicted in Figure 13(a). Conversely, Figures 13(b) and 13(c) clearly show a power concentration in the first symbols, and very low power level elsewhere. In particular, the power distribution in Figure 13(c) is so tight that the transmit symbols assume an impulsive behavior. Typically RF circuitry

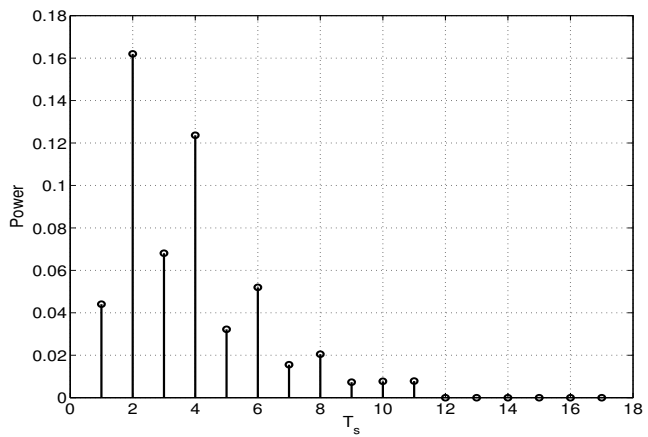

(a) Exponential, slow decay, $\frac{T_{s}}{\tau}=0.75$

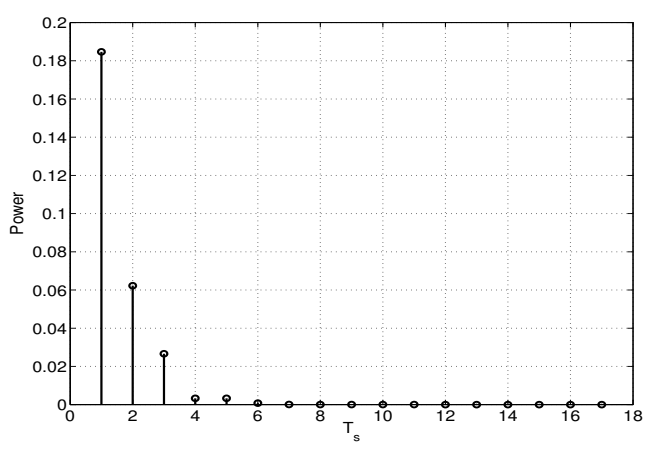

(b) Exponential, fast decay, $\frac{T_{s}}{\tau}=2.5$

Figure 14. PDP of the secondary link.

at the modulators imposes limits on the precision of the generation of very impulsive signal, nonetheless at present we want to understand what would be the outcome of the transmission of such a signal in our scenario. Therefore, we assume that a signal as depicted in Figures 13(b) and 13(c), i.e., its digital representation in our simulations, is obtainable in practice. In the considered scenario the two systems are deployed into the same area hence we can safely assume that $\mathbf{h}_{\mathrm{p}}$ and $\mathbf{h}_{\mathrm{s}}$ may be drawn from the same distribution.

Several sources of attenuation can decrease the received power in wireless communications, e.g., path loss and/or shadowing, thus the corresponding channel is unlikely to be characterized by a uniform PDP. In real applications the channels will most likely fall into the categories represented by the two latter channel snapshots, i.e., exponential PDP with smaller delay spread. Therefore, we focus on this case and analyze both fast and slow decay, considering the uniform PDP case as a benchmark for the other two. Given the precoder computed above, we generate two snapshots of $\mathbf{h}^{(22)}$, as depicted in Figures 14(a) and 14(b).

As previously described, we consider the transmission of the two symbols whose power profile is depicted in Figures 13(a) and 13(b), assuming channels as in Figures 14(a) and 14(b), respectively. In compliance to the OFDM receiving chain, the cyclic prefix is removed from the received signals, shown in Figures 15(a) and 15(b), where the dashed lines represent the transmit signals, plotted for comparison purposes. A huge power penalty can be spotted in both cases, more evident as the delay spread decreases. We remark 


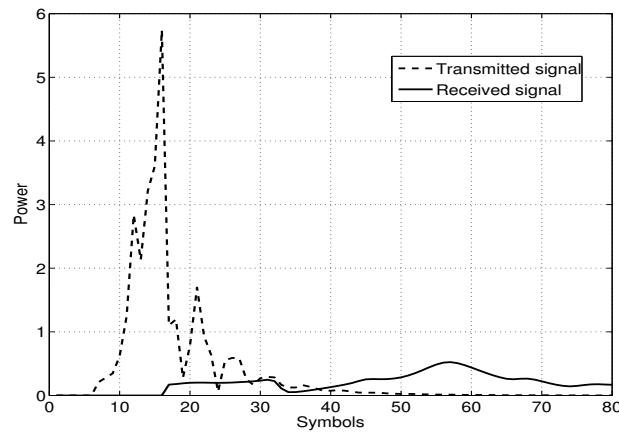

(a) Exponential PDP, slow decay, $\frac{T_{s}}{\tau}=0.75$

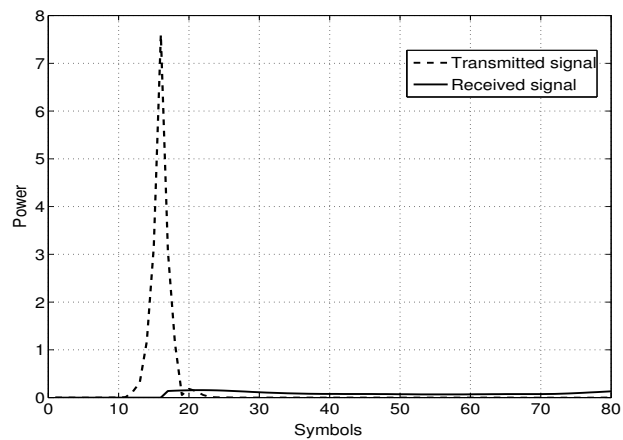

(b) Exponential PDP, fast decay $\frac{T_{s}}{\tau}=2.5$

Figure 15. Transmitted and received signal.

that this is not related to a particular matching between the computed precoder and the faced channel, because by construction $\mathbf{h}^{(21)}$ and $\mathbf{h}^{(22)}$ are independent, hence $\operatorname{ker} \mathcal{T}\left(\mathbf{h}^{(21)}\right)$ and $\operatorname{ker} \mathcal{T}\left(\mathbf{h}^{(22)}\right)$ are distinct. The aforementioned power penalty is strictly related to the nature of the channel, i.e., characterized by short delay spread, hence the largest amount of received power is concentrated in the portion of the signal corresponding to the cyclic prefix. It is important to note that the white gaussian noise added at the receiver does not experience the same penalty, and its statistical properties are not modified by the cyclic prefix removal process. As a consequence, the effective SNR of the resulting $N$ symbols could be significantly different from the SNR before the cyclic prefix removal. Such an SNR drop affects mainly the quality of the following decoding process, thus the achievable rate using this scheme in the considered scenario. In the following section we will investigate this aspect, focusing on the difference of achievable rate between VFDM and an alternative scheme not including a cyclic prefix removal step, i.e., $\mathcal{T}\left(\mathbf{h}^{(22)}\right) \in \mathbb{C}^{(N+L) \times(N+L)}$ circulant matrix.

\subsection{Cyclic Prefix Removal Impact}

As previously seen, the amount of power present in the cyclic prefix is a fundamental characteristic of the received signal. The more the power, the lower the SNR experienced by the resulting $N$ symbols. For the sake of completeness, we consider the whole set of PDP models introduced in Section 8.1. The goal of the following analysis is to understand what would be the achievable

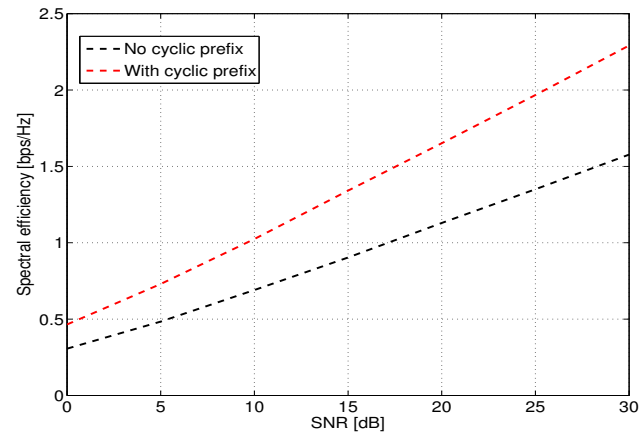

(a) Uniform PDP

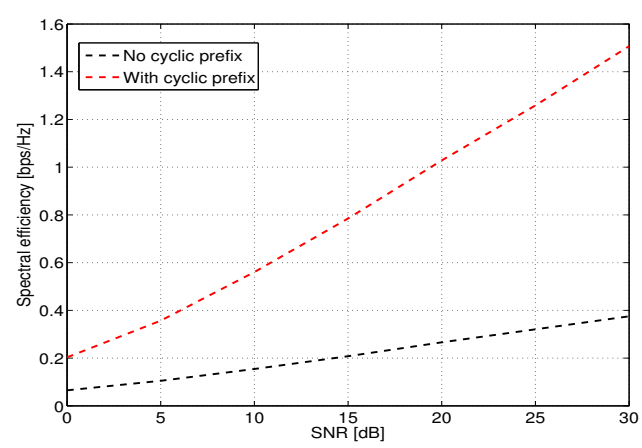

(b) Exponential PDP, slow decay, $\frac{T_{s}}{\tau}=0.75$

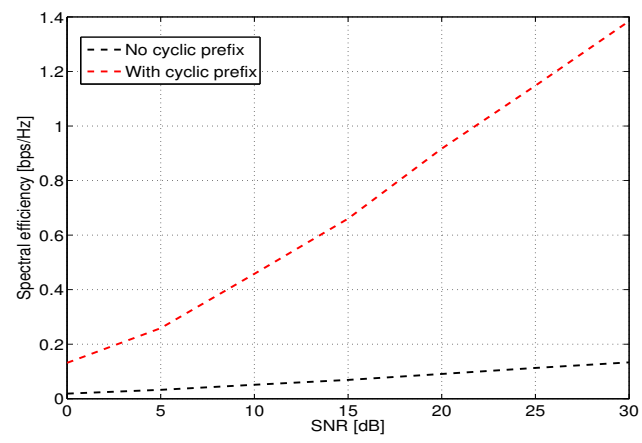

(c) Exponential PDP, fast decay $\frac{T_{S}}{\tau}=2.5$

Figure 16. Achievable rate in case of cyclic prefix decoding and cyclic prefix removal.

rate increase if the receiver could consider the cyclic prefix as a useful portion of the received signal.

We consider channel snapshots and transmit symbols presented in the previous section. We focus on $R X 2$ and, for each one of the considered PDPs, we compute the achievable rate with and without the cyclic prefix removal and we show the performance comparison in Figure 16. Note that, the black curve represents the achievable rate adopting legacy VFDM, whereas the red one shows the achievable one when the cyclic prefix is not discarded. We start from the uniform PDP case, i.e., Figure 16(a). When the cyclic prefix is not removed the achievable rate increases by more than $40 \%$ for both low and high SNR, yielding an SNR gain of $5 \mathrm{~dB}$ and $10 \mathrm{~dB}$ for the two regimes respectively. Even though non negligible, this increment does not change the order of magnitude of the results. In this case, a tradeoff most likely should be found, in order to decide 
which strategy suits the system's needs the most. The increased complexity yields higher costs, but at the same time provides better performance. Depending on the network requirements and quality of service (QoS) constraints, one solution could be preferable to the other and the network designer should frame the receiver architecture accordingly.

In Figure 16(b), an exponential PDP with slow decay is considered. In this case, when the cyclic prefix is not discarded, a gain of more than $200 \%$ and $250 \%$ is achievable for low and high SNR respectively, whereas the SNR gain is more than $20 \mathrm{~dB}$. The power penalty paid after the cyclic prefix removal operation is significant even if it does not alter the order of magnitude of the two results. If we consider the uniform PDP case we notice that, the rate loss when the PDP is exponentially decreasing with slow decay is around $75 \%$ of the achievable rate for the uniform case, for legacy VFDM. On the contrary, if the receiver decodes all the $N+L$ received symbols, the rate loss is less than $35 \%$. The importance of the information stored inside the cyclic prefix, when the channel is characterized by a short delay spread, is now clear and suggests that legacy VFDM is not efficient is this scenario. However, even though neither impressive nor sufficient for the current traffic demands, a spectral efficiency of $0.4 \mathrm{bit} / \mathrm{s} / \mathrm{Hz}$ is what is achieved when adopting third generation standards such as the enhanced data rates for global evolution (EDGE). We could argue that, considered the complete bandwidth sharing with the primary system realized by VFDM, such a spectral efficiency is still an interesting and relevant gain over the standard non cognitive approach. On the other hand, this result is obtained for $\mathrm{SNR}=30 \mathrm{~dB}$, whereas for more practical operating values, i.e., $\mathrm{SNR}=[10,15] \mathrm{dB}$, the spectral efficiency falls to $[0.15,0.2] \mathrm{bit} / \mathrm{s} / \mathrm{Hz}$, mainly useful for voice applications. Naturally, the spectral efficiency of the transmission could be improved significantly with an architectural change at the receiver. Therefore, depending on the requested application and consequent QoS requirements, an architectural change could be advisable.

Finally, in Figure 16(c), we consider an exponential PDP with fast decay. If the receiver does not discard the cyclic prefix, a gain of around $500 \%$ and $1000 \%$ is achievable for low and high SNR respectively. Once more, the SNR gain is extremely large, i.e., more than $30 \mathrm{~dB}$. In this case, the power penalty paid after the cyclic prefix removal operation is so relevant that the performance of the system is strongly affected, hence the order of magnitude of the two results is definitely different. As before, if we compare the current result for legacy VFDM with the uniform PDP case, we see that the rate loss for exponentially decreasing PDP with fast decay is more than $90 \%$ of the achievable rate for the uniform case. This loss is limited to $38 \%$ if the receiver can decode all the $N+L$ symbols, showing the increasing concentration of useful information inside the first $L$ symbols. For this class of fast decaying channels, the legacy VFDM receiver architecture limits the spectral efficiency of the transmission to less than $0.1 \mathrm{bit} / \mathrm{s} / \mathrm{Hz}$ for $\mathrm{SNR}=[10,15] \mathrm{dB}$, result not compliant with the data demands of any future $4^{\text {th }}$ generation device. It is important to note that the rate loss of the legacy VFDM with respect to the modified architecture will be even bigger in case of channels dominated by the LOS component, i.e., Rician fading, typically present in short-range or micro-cellular communications. As a consequence, the spectral efficiency achievable gain brought by the cyclic prefix decoding is fundamental in the VFDM receiver design. To provide a reliable cognitive-based solution based on VFDM, we can not ignore the operating scenario, and the flexibility of this scheme highly depends on the possibility to deal with several different channel statistics and conditions.

\section{Conclusions}

This contribution shows how a VFDM transceiver can be designed and implemented and gives the first proof of concept of a working standalone VFDM transmission. The performed tests show that we can not use the preamble SNR to have insights on payload SNR. Furthermore, a PAPR problem in the payload has been shown and a significant BER detriment w.r.t. to the theoretical results provided in [1] is obtained. A thorough analysis is performed to better understand the structure of the null-space precoder for short r.m.s. delay spread and non-uniform PDP profile. The obtained results enlighten the strong connection between the cyclic prefix removal operation and the spectral efficiency loss experienced by VFDM in practical applications. Consequently, a careful analysis of the VFDM receiver architecture is needed, in order to find adequate and fruitful solutions to adaptively change the cyclic prefix handling strategy, and optimize the achievable performance. Numerical results reinforce this intuition and show a significant spectral efficiency enhancement, yielding results that approach the theoretical bounds and providing useful insights for future improvement of the VFDM test-bed. To this goal, techniques to cope with the PAPR problem, and a more precise procedure to compute the payload SNR will be studied and implemented. Despite the enlightened issues, the obtained results are encouraging and pose the basis for the future implementation of a complete cognitive VFDM based system, using the flexible SDR4All platform.

\section{ACKNOWLEDGMENT}

This work is partially funded by CAPES-COFECUB, Fatih University scientific research fund under project number P50061002 2 (1353), by Alcatel-Lucent within the Alcatel-Lucent Chair on Flexible Radio, Supélec, by Vietnam National University Hanoi under project number TRIG.B, and by the Ministry of Science and Technology of Vietnam under project number 39/2012/HĐ/NĐT. 


$$
\begin{aligned}
\mathbf{v}_{1}= & {\left[w_{1},-b_{41}\left(\frac{h_{\mathrm{p} 1}}{h_{\mathrm{p} 2}}\right)^{3}, b_{41}\left(\frac{h_{\mathrm{p} 1}}{h_{\mathrm{p} 2}}\right)^{2},-b_{41}\left(\frac{h_{\mathrm{p} 1}}{h_{\mathrm{p} 2}}\right), b_{41},-b_{41}\left(\frac{h_{\mathrm{p} 2}}{h_{\mathrm{p} 1}}\right)\right]^{\mathrm{T}} } \\
\mathbf{v}_{2}= & {\left[t_{1}, b_{42}\left(\frac{h_{\mathrm{p} 1}}{h_{\mathrm{p} 2}}\right)^{3}, b_{42}\left(\frac{h_{\mathrm{p} 1}}{h_{\mathrm{p} 2}}\right)^{2},-b_{42}\left(\frac{h_{\mathrm{p} 1}}{h_{\mathrm{p} 2}}\right), b_{42},-b_{42}\left(\frac{h_{\mathrm{p} 2}}{h_{\mathrm{p} 1}}\right)\right]^{\mathrm{T}} } \\
\mathbf{e}_{1}= & \frac{1}{\sqrt{D_{1}}}\left[w_{1},-b_{41}\left(\frac{h_{\mathrm{p} 1}}{h_{\mathrm{p} 2}}\right)^{3}, b_{41}\left(\frac{h_{\mathrm{p} 1}}{h_{\mathrm{p} 2}}\right)^{2},-b_{41}\left(\frac{h_{\mathrm{p} 1}}{h_{\mathrm{p} 2}}\right), b_{41},-b_{41}\left(\frac{h_{\mathrm{p} 2}}{h_{\mathrm{p} 1}}\right)\right]^{\mathrm{T}} \\
\mathbf{e}_{2}= & \frac{1}{\sqrt{D_{3}}}\left[t_{1}-\frac{D_{2} w_{1}}{D_{1}},\left(\frac{h_{\mathrm{p} 1}}{h_{\mathrm{p} 2}}\right)^{3}\left(\frac{b_{41} D_{2}}{D_{1}}-b_{42}\right),\left(\frac{h_{\mathrm{p} 1}}{h_{\mathrm{p} 2}}\right)^{2}\left(b_{42}-\frac{b_{41} D_{2}}{D_{1}}\right),\right. \\
D_{1}= & \left.\left.\mid \frac{h_{\mathrm{p} 1}}{h_{\mathrm{p} 2}}\right)\left(\frac{b_{41} D_{2}}{D_{1}}-\mid b_{42}\right), b_{42}-\frac{b_{41} D_{2}}{D_{1}},\left(\frac{h_{\mathrm{p} 2}}{h_{\mathrm{p} 1}}\right)\left(\frac{b_{41} D_{2}}{D_{1}}-b_{42}\right)\right]^{\mathrm{T}}\left[\left|\frac{h_{\mathrm{p} 1}}{h_{\mathrm{p} 2}}\right|^{6}+\left|\frac{h_{\mathrm{p} 1}}{h_{\mathrm{p} 2}}\right|^{4}+\left|\frac{h_{\mathrm{p} 1}}{h_{\mathrm{p} 2}}\right|^{2}+\left|\frac{h_{\mathrm{p} 1}}{h_{\mathrm{p} 2}}\right|^{-2}+1\right] \\
D_{2}= & t_{1} w_{1}^{*}+b_{42} b_{41}^{*}\left[\left|\frac{h_{\mathrm{p} 1}}{h_{\mathrm{p} 2}}\right|^{6}+\left|\frac{h_{\mathrm{p} 1}}{h_{\mathrm{p} 2}}\right|^{4}+\left|\frac{h_{\mathrm{p} 1}}{h_{\mathrm{p} 2}}\right|^{2}+\left|\frac{h_{\mathrm{p} 1}}{h_{\mathrm{p} 2}}\right|^{-2}+1\right] \\
D_{3}= & \left|t_{1}-\frac{D_{2} w_{1}}{D_{1}}\right|^{2}+\left|\left(\frac{b_{41} D_{2}}{D_{1}}-b_{42}\right)\right|^{2}\left[\left|\frac{h_{\mathrm{p} 1}}{h_{\mathrm{p} 2}}\right|^{6}+\left|\frac{h_{\mathrm{p} 1}}{h_{\mathrm{p} 2}}\right|^{4}+\left|\frac{h_{\mathrm{p} 1}}{h_{\mathrm{p} 2}}\right|^{2}+\left|\frac{h_{\mathrm{p} 2}}{h_{\mathrm{p} 1}}\right|^{-2}+1\right]
\end{aligned}
$$

\section{Appendix}

In the following, the explicit computation of the nullspace precoder columns for $L \neq l$ and $L=L>1$ is presented. When $L=l+1$, the precoder $\mathbf{V}$ is given by $\mathbf{v}_{1}$ and $\mathbf{v}_{2}$ in (32) and (33), where $w_{i}, t_{i} \in \mathbb{C}$, for all $i \in \mathbb{N}$. Its orthonormalized version is given by $\mathbf{e}_{1}$ and $\mathbf{e}_{2}$ in (34), (35), where $D_{1}, D_{2}$ and $D_{3}$ are given in (36), (37) and (38). When $L=l+2, \mathbf{V}$ is given by $\mathbf{v}_{1}, \mathbf{v}_{2}$ and $\mathbf{v}_{3}$, in (39), in (40) and (41), where $s_{i} \in \mathbb{C}$, for all $i \in \mathbb{N}$. Its orthonormalized version is given by $\mathbf{e}_{1}, \mathbf{e}_{2}$ and $\mathbf{e}_{3}$ in (42), (43) and (44), where $D_{1}, D_{2}, D_{3}, D_{5}$ and $D_{6}$ are given in (45), (46), (47), (48), (49) and (50).

Now we consider a channel with three paths, including the LOS, i.e., $l=2$. Let $\mathbf{h}_{(21)}=\left[h_{\mathrm{p} 1}, h_{\mathrm{p} 2}, h_{\mathrm{p} 3}\right]$ and $\mathbf{h}_{\mathrm{s}}=\left[h_{\mathrm{s} 1}, h_{\mathrm{s} 2}, h_{\mathrm{s} 2}\right]$ be the primary and secondary system channel respectively. The matrices $\mathbf{H}_{\mathrm{p}}, \mathbf{H}_{\mathrm{s}}$ are built according to the scheme presented previously. As before we consider different values for $L$. When $L=l, \mathbf{V}$ is given by $\mathbf{v}_{1}$ and $\mathbf{v}_{2}$, in (51) and (52). Its orthonormalized version is given by $\mathbf{e}_{1}$ and $\mathbf{e}_{2}$ in (53), and (54), where $D_{1}, D_{2}$ and $D_{3}$ are given in (55), (56) and (57). When $L=l+1, \mathbf{V}$ is given by $\mathbf{v}_{1}, \mathbf{v}_{2}$ and $\mathbf{v}_{3}$, in (58), in (59) and (60). Its orthonormalized version is given by $\mathbf{e}_{1}, \mathbf{e}_{2}$ and $\mathbf{e}_{3}$ in (61), (62) and (63), where $D_{1}$, $D_{2}, D_{3}, D_{5}$ and $D_{6}$ are given in (64), (65), (66), (67), (68) and (69).

\section{REFERENCES}

[1] L. S. Cardoso, F. R. P. Cavalcanti, M. Kobayashi, and M. Debbah, "Vandermonde-subspace frequency division multiplexing receiver analysis," in IEEE 21st Int. Symp. Personal Indoor and Mobile Radio Communications (PIMRC), 2010, pp. 293-298.

[2] L. S. Cardoso, M. Maso, M. Kobayashi, and M. Debbah, "Orthogonal LTE two-tier cellular networks," in IEEE Int. Conf. Communications (ICC), 5-9 June 2011, pp. 1-5.
[3] R. Bhagavatula and R. W. Heath, "Adaptive limited feedback for sum-rate maximizing beamforming in cooperative multicell systems," IEEE Transactions on Signal Processing, vol. 59, no. 2, pp. 800-811, Feb. 2011.

[4] S. Akoum, M. Zwingelstein-Colin, R. W. Heath, and M. Debbah, "Cognitive cooperation for the downlink of frequency reuse small cells," EURASIP Journal on Advances in Signal Processing, vol. 2011, 2011.

[5] J.-M. Wu, T.-F. Yang, and H.-J. Chou, "MIMO active interference alignment for underlay cognitive radio," in IEEE Int. Conf. Communications (ICC), 23-27 May 2010, pp. 1-5.

[6] Sdr4all. [Online] http://www.flexible-radio.com/sdr4all.

[7] 3GPP, Physical Layer Aspects for Evolved UTRA, 3GPP Std. TR 25.814, Rev. 2.0.0, 2006.

[8] J. Mitola, "Cognitive Radio An Integrated Agent Architecture for Software Defined Radio," Ph.D. dissertation, Royal Institute of Technology (KTH), May 2000.

[9] A. Ghasemi and E. S. Sousa, "Fundamental limits of spectrumsharing in fading environments," IEEE Transactions on Wireless Communications, vol. 6, no. 2, pp. 649-658, Feb. 2007.

[10] S. Srinivasa and S. A. Jafar, "The throughput potential of cognitive radio: A theoretical perspective," in 40th Asilomar Conference on Signals, Systems and Computers, 2006, pp. 221-225.

[11] L. S. Cardoso, M. Kobayashi, Ø. Ryan, and M. Debbah, "Vandermonde frequency division multiplexing for cognitive radio," in 9th IEEE Workshop on Signal Processing Advances in Wireless Communications (SPAWC), 6-9 July 2008, pp. 421-425.

[12] G. Golub and C. Van Loan, Matrix Computations. Johns Hopkins University Press, 1996.

[13] M. Kobayashi, M. Debbah, and S. Shamai, "Secured communication over frequency-selective fading channels: A practical Vandermonde precoding," EURASIPJournal on Wireless Communications and Networking, 2009.

[14] Tools4sdr. [Online] http://www.flexible-radio.com/tools4sdr.

[15] F. A. Hamza. The USRP under $1.5 \times$ magnifying lens! [Online] https://microembedded.googlecode.com/files/ USRP_Documentation.pdf.

[16] M. Golay, "Complementary series," IRE Transactions on Information Theory, vol. IT-7, p. 82-87, 1961.

[17] Wireless LAN medium access control (MAC) and physical (PHY) layer specifications- high speed physical layer in 5GHz band. standards.ieee.org/getieee802/download/802.11a1999.pdf.

[18] ETSI, Broadband Radio Access Networks (BRAN); HIPERLAN Type 2; Physical (PHY) layer, 2002 Std. TS 101475 , Rev. v1.2.2.

[19] D. Tse and P. Viswanath, Fundamentals of Wireless Communication. Cambridge University Press, 2005. 


$$
\begin{aligned}
\mathbf{v}_{1}= & {\left[w_{1},-b_{41}\left(\frac{h_{\mathrm{p} 1}}{h_{\mathrm{p} 2}}\right)^{3}, b_{41}\left(\frac{h_{\mathrm{p} 1}}{h_{\mathrm{p} 2}}\right)^{2},-b_{41}\left(\frac{h_{\mathrm{p} 1}}{h_{\mathrm{p} 2}}\right), b_{41},-b_{41}\left(\frac{h_{\mathrm{p} 2}}{h_{\mathrm{p} 1}}\right)\right]^{\mathrm{T}} } \\
\mathbf{v}_{2}= & {\left[t_{1}, b_{42}\left(\frac{h_{\mathrm{p} 1}}{h_{\mathrm{p} 2}}\right)^{3}, b_{42}\left(\frac{h_{\mathrm{p} 1}}{h_{\mathrm{p} 2}}\right)^{2},-b_{42}\left(\frac{h_{\mathrm{p} 1}}{h_{\mathrm{p} 2}}\right), b_{42},-b_{42}\left(\frac{h_{\mathrm{p} 2}}{h_{\mathrm{p} 1}}\right)\right]^{\mathrm{T}} } \\
\mathbf{e}_{1}= & \frac{1}{\sqrt{D_{1}}}\left[w_{1},-b_{41}\left(\frac{h_{\mathrm{p} 1}}{h_{\mathrm{p} 2}}\right)^{3}, b_{41}\left(\frac{h_{\mathrm{p} 1}}{h_{\mathrm{p} 2}}\right)^{2},-b_{41}\left(\frac{h_{\mathrm{p} 1}}{h_{\mathrm{p} 2}}\right), b_{41},-b_{41}\left(\frac{h_{\mathrm{p} 2}}{h_{\mathrm{p} 1}}\right)\right]^{\mathrm{T}} \\
\mathbf{e}_{2}= & \frac{1}{\sqrt{D_{3}}}\left[t_{1}-\frac{D_{2} w_{1}}{D_{1}},\left(\frac{h_{\mathrm{p} 1}}{h_{\mathrm{p} 2}}\right)^{3}\left(\frac{b_{41} D_{2}}{D_{1}}-b_{42}\right),\left(\frac{h_{\mathrm{p} 1}}{h_{\mathrm{p} 2}}\right)^{2}\left(b_{42}-\frac{b_{41} D_{2}}{D_{1}}\right),\right. \\
& \left.\left(\frac{h_{\mathrm{p} 1}}{h_{\mathrm{p} 2}}\right)\left(\frac{b_{41} D_{2}}{D_{1}}-b_{42}\right), b_{42}-\frac{b_{41} D_{2}}{D_{1}},\left(\frac{h_{\mathrm{p} 2}}{h_{\mathrm{p} 1}}\right)\left(\frac{b_{41} D_{2}}{D_{1}}-b_{42}\right)\right]^{\mathrm{T}}
\end{aligned}
$$

$D_{1}=\left|w_{1}\right|^{2}+\left|b_{41}\right|^{2}\left[\left|\frac{h_{\mathrm{p} 1}}{h_{\mathrm{p} 2}}\right|^{6}+\left|\frac{h_{\mathrm{p} 1}}{h_{\mathrm{p} 2}}\right|^{4}+\left|\frac{h_{\mathrm{p} 1}}{h_{\mathrm{p} 2}}\right|^{2}+\left|\frac{h_{\mathrm{p} 1}}{h_{\mathrm{p} 2}}\right|^{-2}+1\right]$

$D_{2}=t_{1} w_{1}^{*}+b_{42} b_{41}^{*}\left[\left|\frac{h_{\mathrm{p} 1}}{h_{\mathrm{p} 2}}\right|^{6}+\left|\frac{h_{\mathrm{p} 1}}{h_{\mathrm{p} 2}}\right|^{4}+\left|\frac{h_{\mathrm{p} 1}}{h_{\mathrm{p} 2}}\right|^{2}+\left|\frac{h_{\mathrm{p} 1}}{h_{\mathrm{p} 2}}\right|^{-2}+1\right]$

$D_{3}=\left|t_{1}-\frac{D_{2} w_{1}}{D_{1}}\right|^{2}+\left|\left(\frac{b_{41} D_{2}}{D_{1}}-b_{42}\right)\right|^{2}\left[\left|\frac{h_{\mathrm{p} 1}}{h_{\mathrm{p} 2}}\right|^{6}+\left|\frac{h_{\mathrm{p} 1}}{h_{\mathrm{p} 2}}\right|^{4}+\left|\frac{h_{\mathrm{p} 1}}{h_{\mathrm{p} 2}}\right|^{2}+\left|\frac{h_{\mathrm{p} 2}}{h_{\mathrm{p} 1}}\right|^{-2}+1\right]$

$$
\begin{aligned}
\mathbf{v}_{1}= & {\left[w_{1}, w_{2},-b_{41}\left(\frac{h_{\mathrm{p} 1}}{h_{\mathrm{p} 2}}\right)^{3}, b_{41}\left(\frac{h_{\mathrm{p} 1}}{h_{\mathrm{p} 2}}\right)^{2},-b_{41}\left(\frac{h_{\mathrm{p} 1}}{h_{\mathrm{p} 2}}\right), b_{41},-b_{41}\left(\frac{h_{\mathrm{p} 2}}{h_{\mathrm{p} 1}}\right)\right]^{\mathrm{T}} } \\
\mathbf{v}_{2}= & {\left[t_{1}, t_{2},-b_{42}\left(\frac{h_{\mathrm{p} 1}}{h_{\mathrm{p} 2}}\right)^{3}, b_{42}\left(\frac{h_{\mathrm{p} 1}}{h_{\mathrm{p} 2}}\right)^{2},-b_{42}\left(\frac{h_{\mathrm{p} 1}}{h_{\mathrm{p} 2}}\right), b_{42},-b_{42}\left(\frac{h_{\mathrm{p} 2}}{h_{\mathrm{p} 1}}\right)\right]^{\mathrm{T}} } \\
\mathbf{v}_{3}= & {\left[s_{1}, s_{2}, b_{43}\left(\frac{h_{\mathrm{p} 1}}{h_{\mathrm{p} 2}}\right)^{3}, b_{43}\left(\frac{h_{\mathrm{p} 1}}{h_{\mathrm{p} 2}}\right)^{2},-b_{43}\left(\frac{h_{\mathrm{p} 1}}{h_{\mathrm{p} 2}}\right), b_{43},-b_{43}\left(\frac{h_{\mathrm{p} 2}}{h_{\mathrm{p} 1}}\right)\right]^{\mathrm{T}} } \\
\mathbf{e}_{1}= & \frac{1}{\sqrt{D_{1}}}\left[w_{1}, w_{2},-b_{41}\left(\frac{h_{\mathrm{p} 1}}{h_{\mathrm{p} 2}}\right)^{3}, b_{41}\left(\frac{h_{\mathrm{p} 1}}{h_{\mathrm{p} 2}}\right)^{2},-b_{41}\left(\frac{h_{\mathrm{p} 1}}{h_{\mathrm{p} 2}}\right), b_{41},-b_{41}\left(\frac{h_{\mathrm{p} 2}}{h_{\mathrm{p} 1}}\right)^{3}\right]^{\mathrm{T}} \\
\mathbf{e}_{2}= & \frac{1}{\sqrt{D_{3}}}\left[t_{1}-\frac{D_{2} w_{1}}{D_{1}}, t_{2}-\frac{D_{2} w_{2}}{D_{1}},\left(\frac{h_{\mathrm{p} 1}}{h_{\mathrm{p} 2}}\right)^{3}\left(\frac{b_{41} D_{2}}{D_{1}}-b_{42}\right),\left(\frac{h_{\mathrm{p} 1}}{h_{\mathrm{p} 2}}\right)^{2}\left(b_{42}-\frac{b_{41} D_{2}}{D_{1}}\right),\left(\frac{h_{\mathrm{p} 1}}{h_{\mathrm{p} 2}}\right)\left(\frac{b_{41} D_{2}}{D_{1}}-b_{42}\right),\right. \\
& \left.b_{42}-\frac{b_{41} D_{2}}{D_{1}},\left(\frac{h_{\mathrm{p} 2}}{h_{\mathrm{p} 1}}\right)\left(\frac{b_{41} D_{2}}{D_{1}}-b_{42}\right)\right]^{\mathrm{T}}
\end{aligned}
$$

$$
\begin{aligned}
\mathbf{e}_{3}= & \frac{1}{\sqrt{D_{6}}}\left[s_{1}-\frac{D_{4} w_{1}}{D_{1}}-\frac{D_{5}\left(t_{1}-\frac{D_{2} w_{1}}{D 1}\right)}{D_{3}}, s_{2}-\frac{D_{4} w_{2}}{D_{1}}-\frac{D_{5}\left(t_{2}-\frac{D_{2} w_{2}}{D 1}\right)}{D_{3}},\right. \\
& \left(\frac{h_{\mathrm{p} 1}}{h_{\mathrm{p} 2}}\right)^{3}\left[\frac{D_{5}}{D_{3}}\left(b_{42}-\frac{b_{41} D_{2}}{D_{1}}\right)+\frac{b_{41} D_{4}}{D_{1}}-b_{43}\right],\left(\frac{h_{\mathrm{p} 1}}{h_{\mathrm{p} 2}}\right)^{2}\left[\frac{D_{5}}{D_{3}}\left(\frac{b_{41} D_{2}}{D_{1}}-b_{42}\right)+b_{43}-\frac{b_{41} D_{4}}{D_{1}}\right], \\
& \left(\frac{h_{\mathrm{p} 1}}{h_{\mathrm{p} 2}}\right)\left[\frac{D_{5}}{D_{3}}\left(b_{42}-\frac{b_{41} D_{2}}{D_{1}}\right)+\frac{b_{41} D_{4}}{D_{1}}-b_{43}\right], \frac{D_{5}}{D_{3}}\left(\frac{b_{41} D_{2}}{D_{1}}-b_{42}\right)+b_{43}-\frac{b_{41} D_{4}}{D_{1}}, \\
& \left.\left(\frac{h_{\mathrm{p} 2}}{h_{\mathrm{p} 1}}\right)\left[\frac{D_{5}}{D_{3}}\left(b_{42}-\frac{b_{41} D_{2}}{D_{1}}\right)+\frac{b_{41} D_{4}}{D_{1}}-b_{43}\right]\right]^{\mathrm{T}}
\end{aligned}
$$




$$
\begin{aligned}
& D_{1}=\left|w_{1}\right|^{2}+\left|w_{2}\right|^{2}+\left|b_{41}\right|^{2}\left(\left|\frac{h_{\mathrm{p} 1}}{h_{\mathrm{p} 2}}\right|^{6}+\left|\frac{h_{\mathrm{p} 1}}{h_{\mathrm{p} 2}}\right|^{4}+\left|\frac{h_{\mathrm{p} 1}}{h_{\mathrm{p} 2}}\right|^{2}+\left|\frac{h_{\mathrm{p} 1}}{h_{\mathrm{p} 2}}\right|^{-2}+1\right) \\
& D_{2}=t_{1} w_{1}^{*}+t_{2} w_{2}^{*}+b_{42} b_{41}^{*}\left(\left|\frac{h_{\mathrm{p} 1}}{h_{\mathrm{p} 2}}\right|^{6}+\left|\frac{h_{\mathrm{p} 1}}{h_{\mathrm{p} 2}}\right|^{4}+\left|\frac{h_{\mathrm{p} 1}}{h_{\mathrm{p} 2}}\right|^{2}+\left|\frac{h_{\mathrm{p} 1}}{h_{\mathrm{p} 2}}\right|^{-2}+1\right) \\
& D_{3}=\left|t_{1}-\frac{D_{2} w_{1}}{D_{1}}\right|^{2}+\left|t_{2}-\frac{D_{2} w_{2}}{D_{1}}\right|^{2}+\left|\left(\frac{b_{41} D_{2}}{D_{1}}-b_{42}\right)\right|^{2}\left[\left|\frac{h_{\mathrm{p} 1}}{h_{\mathrm{p} 2}}\right|^{6}+\left|\frac{h_{\mathrm{p} 1}}{h_{\mathrm{p} 2}}\right|^{4}+\left|\frac{h_{\mathrm{p} 1}}{h_{\mathrm{p} 2}}\right|^{2}+\left|\frac{h_{\mathrm{p} 2}}{h_{\mathrm{p} 1}}\right|^{-2}+1\right] \\
& D_{4}=s_{1} w_{1}^{*}+s_{2} w_{2}^{*}+b_{43} b_{41}^{*}\left(\left|\frac{h_{\mathrm{p} 1}}{h_{\mathrm{p} 2}}\right|^{6}+\left|\frac{h_{\mathrm{p} 1}}{h_{\mathrm{p} 2}}\right|^{4}+\left|\frac{h_{\mathrm{p} 1}}{h_{\mathrm{p} 2}}\right|^{2}+\left|\frac{h_{\mathrm{p} 1}}{h_{\mathrm{p} 2}}\right|^{-2}+1\right) \\
& D_{5}=s_{1}\left(t_{1}^{*}-\frac{D_{2}^{*} w_{1}^{*}}{D_{1}}\right)+s_{2}\left(t_{2}^{*}-\frac{D_{2}^{*} w_{2} *}{D_{1}}\right)+b_{43}\left(b_{42}^{*}-\frac{D_{2}^{*} b_{41}^{*}}{D_{1}}\right)\left(\left|\frac{h_{\mathrm{p} 1}}{h_{\mathrm{p} 2}}\right|^{6}+\left|\frac{h_{\mathrm{p} 1}}{h_{\mathrm{p} 2}}\right|^{4}+\left|\frac{h_{\mathrm{p} 1}}{h_{\mathrm{p} 2}}\right|^{2}+\left|\frac{h_{\mathrm{p} 1}}{h_{\mathrm{p} 2}}\right|^{-2}+1\right) \\
& D_{6}=\left|s_{1}-\frac{D_{4} w_{1}}{D_{1}}-\frac{D_{5}\left(t_{1}-\frac{D_{2} w_{1}}{D_{1}}\right)}{D_{3}}\right|^{2}+\left|s_{2}-\frac{D_{4} w_{2}}{D_{1}}-\frac{D_{5}\left(t_{2}-\frac{D_{2} w_{2}}{D_{1}}\right)}{D_{3}}\right|^{2}+ \\
& +\left|\frac{D_{5}}{D_{3}}\left(b_{42}-\frac{b_{41} D_{2}}{D_{1}}\right)+\frac{b_{41} D_{4}}{D_{1}}-b_{43}\right|^{2}\left[\left|\frac{h_{\mathrm{p} 1}}{h_{\mathrm{p} 2}}\right|^{6}+\left|\frac{h_{\mathrm{p} 1}}{h_{\mathrm{p} 2}}\right|^{4}+\left|\frac{h_{\mathrm{p} 1}}{h_{\mathrm{p} 2}}\right|^{2}+\left|\frac{h_{\mathrm{p} 1}}{h_{\mathrm{p} 2}}\right|^{-2}+1\right] \\
& \mathbf{v}_{1}=\left[\frac{1}{h_{\mathrm{p} 3}^{3}}\left[b_{51}\left(h_{\mathrm{p} 1} h_{\mathrm{p} 2}^{2}-h_{\mathrm{p} 1}^{2} h_{\mathrm{p} 3}\right)-b_{41}\left(h_{\mathrm{p} 2}^{3}-2 h_{\mathrm{p} 1} h_{\mathrm{p} 2} h_{\mathrm{p} 3}\right)\right], \frac{1}{h_{\mathrm{p} 3}^{2}}\left[b_{51} h_{\mathrm{p} 1} h_{\mathrm{p} 2}+b_{41}\left(h_{\mathrm{p} 2}^{2}-h_{\mathrm{p} 1} h_{\mathrm{p} 3}\right)\right],\right. \\
& \left.-\frac{1}{h_{\mathrm{p} 3}}\left(b_{51} h_{\mathrm{p} 1}+b_{41} h_{\mathrm{p} 2}\right), b_{41}, b_{51},-\frac{1}{h_{\mathrm{p} 1}}\left(b_{51} h_{\mathrm{p} 2}+b_{41} h_{\mathrm{p} 3}\right)\right]^{\mathrm{T}} \\
& \mathbf{v}_{2}=\left[\frac{1}{h_{\mathrm{p} 3}^{3}}\left[b_{52}\left(h_{\mathrm{p} 1} h_{\mathrm{p} 2}^{2}-h_{\mathrm{p} 1}^{2} h_{\mathrm{p} 3}\right)-b_{42}\left(h_{\mathrm{p} 2}^{3}-2 h_{\mathrm{p} 1} h_{\mathrm{p} 2} h_{\mathrm{p} 3}\right)\right], \frac{1}{h_{\mathrm{p} 3}^{2}}\left[b_{52} h_{\mathrm{p} 1} h_{\mathrm{p} 2}+b_{42}\left(h_{\mathrm{p} 2}^{2}-h_{\mathrm{p} 1} h_{\mathrm{p} 3}\right)\right],\right. \\
& \left.-\frac{1}{h_{\mathrm{p} 3}}\left(b_{52} h_{\mathrm{p} 1}+b_{41} h_{\mathrm{p} 2}\right), b_{42}, b_{52},-\frac{1}{h_{\mathrm{p} 1}}\left(b_{52} h_{\mathrm{p} 2}+b_{42} h_{\mathrm{p} 3}\right)\right]^{\mathrm{T}} \\
& \mathbf{e}_{1}=\frac{1}{\sqrt{D_{1}}}\left[\frac{1}{h_{\mathrm{p} 3}^{3}}\left[b_{51}\left(h_{\mathrm{p} 1} h_{\mathrm{p} 2}^{2}-h_{\mathrm{p} 1}^{2} h_{\mathrm{p} 3}\right)-b_{41}\left(h_{\mathrm{p} 2}^{3}-2 h_{\mathrm{p} 1} h_{\mathrm{p} 2} h_{\mathrm{p} 3}\right)\right], \frac{1}{h_{\mathrm{p} 3}^{2}}\left[b_{51} h_{\mathrm{p} 1} h_{\mathrm{p} 2}+b_{41}\left(h_{\mathrm{p} 2}^{2}-h_{\mathrm{p} 1} h_{\mathrm{p} 3}\right)\right],\right. \\
& \left.-\frac{1}{h_{\mathrm{p} 3}}\left(b_{51} h_{\mathrm{p} 1}+b_{41} h_{\mathrm{p} 2}\right), b_{41}, b_{51},-\frac{1}{h_{\mathrm{p} 1}}\left(b_{51} h_{\mathrm{p} 2}+b_{41} h_{\mathrm{p} 3}\right)\right]^{\mathrm{T}} \\
& \mathbf{e}_{2}=\frac{1}{\sqrt{D_{3}}}\left[-\frac{1}{h_{\mathrm{p} 3}^{3}}\left(\left(\frac{D_{2} b_{51}}{D_{1}}-b_{52}\right)\left(h_{\mathrm{p} 1} h_{\mathrm{p} 2}^{2}-h_{\mathrm{p} 1}^{2} h_{\mathrm{p} 3}\right)-\left(\frac{D_{2} b_{41}}{D_{1}}-b_{42}\right)\left(h_{\mathrm{p} 2}^{3}-2 h_{\mathrm{p} 1} h_{\mathrm{p} 2} h_{\mathrm{p} 3}\right)\right),\right. \\
& -\frac{1}{h_{\mathrm{p} 3}^{2}}\left(\left(\frac{D_{2} b_{51}}{D_{1}}-b_{52}\right) h_{\mathrm{p} 1} h_{\mathrm{p} 2}+\left(\frac{D_{2} b_{41}}{D_{1}}-b_{42}\right)\left(h_{\mathrm{p} 2}^{2}-h_{\mathrm{p} 1} h_{\mathrm{p} 3}\right)\right) \text {, } \\
& \frac{1}{h_{\mathrm{p} 3}}\left(\left(\frac{D_{2} b_{51}}{D_{1}}-b_{52}\right) h_{\mathrm{p} 1}+\left(\frac{D_{2} b_{41}}{D_{1}}-b_{42}\right) h_{\mathrm{p} 2}\right), b_{42}-\frac{b_{41} D_{2}}{D_{1}}, b_{52}-\frac{b_{51} D_{2}}{D_{1}}, \\
& \left.\frac{1}{h_{\mathrm{p} 1}}\left(\left(\frac{D_{2} b_{51}}{D_{1}}-b_{52}\right) h_{\mathrm{p} 2}+\left(\frac{D_{2} b_{41}}{D_{1}}-b_{42}\right) h_{\mathrm{p} 3}\right)\right]^{\mathrm{T}} \\
& D_{1}=\left|b_{41}\right|^{2}+\left|b_{51}\right|^{2}+\left|\frac{b_{41} h_{\mathrm{p} 3}+b_{51} h_{\mathrm{p} 2}}{h_{\mathrm{p} 1}}\right|^{2}+\left|\frac{b_{51} h_{\mathrm{p} 1}+b_{41} h_{\mathrm{p} 2}}{h_{\mathrm{p} 3}}\right|^{2}+\left|\frac{b_{51} h_{\mathrm{p} 1} h_{\mathrm{p} 2}+b_{41}\left(h_{\mathrm{p} 2}^{2}-h_{\mathrm{p} 1} h_{\mathrm{p} 3}\right)}{h_{\mathrm{p} 3}^{2}}\right|^{2}+ \\
& +\left|\frac{b_{51}\left(h_{\mathrm{p} 1} h_{\mathrm{p} 2}^{2}-h_{\mathrm{p} 1}^{2} h_{\mathrm{p} 3}\right)-b_{41}\left(h_{\mathrm{p} 2}^{3}-2 h_{\mathrm{p} 1} h_{\mathrm{p} 2} h_{\mathrm{p} 3}\right)}{h_{\mathrm{p} 3}^{3}}\right|^{2}
\end{aligned}
$$




$$
\begin{aligned}
& D_{2}=b_{42} b_{41}^{*}+b_{52} b_{51}^{*}+\left|\frac{1}{h_{\mathrm{p} 1}}\right|^{2}\left[\left(b_{42} h_{\mathrm{p} 3}+b_{52} h_{\mathrm{p} 2}\right)\left(b_{41} h_{\mathrm{p} 3}+b_{51} h_{\mathrm{p} 2}\right)^{*}\right] \\
& +\left|\frac{1}{h_{\mathrm{p} 3}}\right|^{2}\left[\left(b_{52} h_{\mathrm{p} 1}+b_{42} h_{\mathrm{p} 2}\right)\left(b_{51} h_{\mathrm{p} 1}+b_{41} h_{\mathrm{p} 2}\right)^{*}\right] \\
& +\left|\frac{1}{h_{\mathrm{p} 3}}\right|^{4}\left[b_{52} h_{\mathrm{p} 1} h_{\mathrm{p} 2}+b_{42}\left(h_{\mathrm{p} 2}^{2}-h_{\mathrm{p} 1} h_{\mathrm{p} 3}\right)\right]\left[b_{51} h_{\mathrm{p} 1} h_{\mathrm{p} 2}+b_{41}\left(h_{\mathrm{p} 2}^{2}-h_{\mathrm{p} 1} h_{\mathrm{p} 3}\right)\right]^{*} \\
& +\left|\frac{1}{h_{\mathrm{p} 3}}\right|^{6}\left[b_{52}\left(h_{\mathrm{p} 1} h_{\mathrm{p} 2}^{2}-h_{\mathrm{p} 1}^{2} h_{\mathrm{p} 3}\right)-b_{42}\left(h_{\mathrm{p} 2}^{3}-2 h_{\mathrm{p} 1} h_{\mathrm{p} 2} h_{\mathrm{p} 3}\right)\right]\left[b_{51}\left(h_{\mathrm{p} 1} h_{\mathrm{p} 2}^{2}-h_{\mathrm{p} 1}^{2} h_{\mathrm{p} 3}\right)-b_{41}\left(h_{\mathrm{p} 2}^{3}-2 h_{\mathrm{p} 1} h_{\mathrm{p} 2} h_{\mathrm{p} 3}\right)\right]^{*} \\
& D_{3}=\left|b_{42}-\frac{b_{41} D_{2}}{D_{1}}\right|^{2}+\left|b_{52}-\frac{b_{51} D_{2}}{D_{1}}\right|^{2}+\left|\frac{1}{h_{\mathrm{p} 1}}\right|^{2}\left|\left(\frac{D_{2} b_{51}}{D_{1}}-b_{52}\right) h_{\mathrm{p} 2}+\left(\frac{D_{2} b_{41}}{D_{1}}-b_{42}\right) h_{\mathrm{p} 3}\right|^{2}+ \\
& +\left|\frac{1}{h_{\mathrm{p} 3}}\right|^{2}\left|\left(\frac{D_{2} b_{51}}{D_{1}}-b_{52}\right) h_{\mathrm{p} 1}+\left(\frac{D_{2} b_{41}}{D_{1}}-b_{42}\right) h_{\mathrm{p} 2}\right|^{2}+ \\
& +\left|\frac{1}{h_{\mathrm{p} 3}}\right|^{4}\left|\left(\frac{D_{2} b_{51}}{D_{1}}-b_{52}\right) h_{\mathrm{p} 1} h_{\mathrm{p} 2}+\left(\frac{D_{2} b_{41}}{D_{1}}-b_{42}\right)\left(h_{\mathrm{p} 2}^{2}-h_{\mathrm{p} 1} h_{\mathrm{p} 3}\right)\right|^{2}+ \\
& +\left|\frac{1}{h_{\mathrm{p} 3}}\right|^{6}\left|\left(\frac{D_{2} b_{51}}{D_{1}}-b_{52}\right)\left(h_{\mathrm{p} 1} h_{\mathrm{p} 2}^{2}-h_{\mathrm{p} 1}^{2} h_{\mathrm{p} 3}\right)-\left(\frac{D_{2} b_{41}}{D_{1}}-b_{42}\right)\left(h_{\mathrm{p} 2}^{3}-2 h_{\mathrm{p} 1} h_{\mathrm{p} 2} h_{\mathrm{p} 3}\right)\right|^{2} \\
& \mathbf{v}_{1}=\left[w_{1}, \frac{1}{h_{\mathrm{p} 3}^{3}}\left(b_{51}\left(h_{\mathrm{p} 1} h_{\mathrm{p} 2}^{2}-h_{\mathrm{p} 1}^{2} h_{\mathrm{p} 3}\right)-b_{41}\left(h_{\mathrm{p} 2}^{3}-2 h_{\mathrm{p} 1} h_{\mathrm{p} 2} h_{\mathrm{p} 3}\right)\right),\right. \\
& \left.\frac{1}{h_{\mathrm{p} 3}^{2}}\left(b_{51} h_{\mathrm{p} 1} h_{\mathrm{p} 2}+b_{41}\left(h_{\mathrm{p} 2}^{2}-h_{\mathrm{p} 1} h_{\mathrm{p} 3}\right)\right),-\frac{1}{h_{\mathrm{p} 3}}\left(b_{51} h_{\mathrm{p} 1}+b_{41} h_{\mathrm{p} 2}\right), b_{41}, b_{51},-\frac{1}{h_{\mathrm{p} 1}}\left(b_{51} h_{\mathrm{p} 2}+b_{41} h_{\mathrm{p} 3}\right)\right]^{\mathrm{T}} \\
& \mathbf{v}_{2}=\left[t_{1}, \frac{1}{h_{\mathrm{p} 3}^{3}}\left(b_{52}\left(h_{\mathrm{p} 1} h_{\mathrm{p} 2}^{2}-h_{\mathrm{p} 1}^{2} h_{\mathrm{p} 3}\right)-b_{42}\left(h_{\mathrm{p} 2}^{3}-2 h_{\mathrm{p} 1} h_{\mathrm{p} 2} h_{\mathrm{p} 3}\right)\right),\right. \\
& \left.\frac{1}{h_{\mathrm{p} 3}^{2}}\left(b_{52} h_{\mathrm{p} 1} h_{\mathrm{p} 2}+b_{42}\left(h_{\mathrm{p} 2}^{2}-h_{\mathrm{p} 1} h_{\mathrm{p} 3}\right)\right),-\frac{1}{h_{\mathrm{p} 3}}\left(b_{52} h_{\mathrm{p} 1}+b_{42} h_{\mathrm{p} 2}\right), b_{42}, b_{52},-\frac{1}{h_{\mathrm{p} 1}}\left(b_{52} h_{\mathrm{p} 2}+b_{42} h_{\mathrm{p} 3}\right)\right]^{\mathrm{T}} \\
& \mathbf{v}_{3}=\left[s_{1}, \frac{1}{h_{\mathrm{p} 3}^{3}}\left(b_{53}\left(h_{\mathrm{p} 1} h_{\mathrm{p} 2}^{2}-h_{\mathrm{p} 1}^{2} h_{\mathrm{p} 3}\right)-b_{43}\left(h_{\mathrm{p} 2}^{3}-2 h_{\mathrm{p} 1} h_{\mathrm{p} 2} h_{\mathrm{p} 3}\right)\right)\right. \text {, } \\
& \left.\frac{1}{h_{\mathrm{p} 3}^{2}}\left(b_{53} h_{\mathrm{p} 1} h_{\mathrm{p} 2}+b_{43}\left(h_{\mathrm{p} 2}^{2}-h_{\mathrm{p} 1} h_{\mathrm{p} 3}\right)\right),-\frac{1}{h_{\mathrm{p} 3}}\left(b_{53} h_{\mathrm{p} 1}+b_{43} h_{\mathrm{p} 2}\right), b_{43}, b_{53},-\frac{1}{h_{\mathrm{p} 1}}\left(b_{53} h_{\mathrm{p} 2}+b_{43} h_{\mathrm{p} 3}\right)\right]^{\mathrm{T}} \\
& \mathbf{e}_{1}=\frac{1}{\sqrt{D_{1}}}\left[w_{1}, \frac{1}{h_{\mathrm{p} 3}^{3}}\left(b_{51}\left(h_{\mathrm{p} 1} h_{\mathrm{p} 2}^{2}-h_{\mathrm{p} 1}^{2} h_{\mathrm{p} 3}\right)-b_{41}\left(h_{\mathrm{p} 2}^{3}-2 h_{\mathrm{p} 1} h_{\mathrm{p} 2} h_{\mathrm{p} 3}\right)\right), \frac{1}{h_{\mathrm{p} 3}^{2}}\left(b_{51} h_{\mathrm{p} 1} h_{\mathrm{p} 2}+b_{41}\left(h_{\mathrm{p} 2}^{2}-h_{\mathrm{p} 1} h_{\mathrm{p} 3}\right)\right),\right. \\
& \left.-\frac{1}{h_{\mathrm{p} 3}}\left(b_{51} h_{\mathrm{p} 1}+b_{41} h_{\mathrm{p} 2}\right), b_{41}, b_{51},-\frac{1}{h_{\mathrm{p} 1}}\left(b_{51} h_{\mathrm{p} 2}+b_{41} h_{\mathrm{p} 3}\right)\right]^{\mathrm{T}} \\
& \mathbf{e}_{2}=\frac{1}{\sqrt{D_{3}}}\left[t_{1}-\frac{D_{2} w_{1}}{D_{1}}, \frac{1}{h_{\mathrm{p} 3}^{3}}\left(\left(\frac{D_{2} b_{41}}{D_{1}}-b_{42}\right)\left(h_{\mathrm{p} 2}^{3}-2 h_{\mathrm{p} 1} h_{\mathrm{p} 2} h_{\mathrm{p} 3}\right)-\left(\frac{D_{2} b_{51}}{D_{1}}-b_{52}\right)\left(h_{\mathrm{p} 1} h_{\mathrm{p} 2}^{2}-h_{\mathrm{p} 1}^{2} h_{\mathrm{p} 3}\right)\right),\right. \\
& -\frac{1}{h_{\mathrm{p} 3}^{2}}\left(\left(\frac{D_{2} b_{41}}{D_{1}}-b_{42}\right)\left(h_{\mathrm{p} 2}^{2}-h_{\mathrm{p} 1} h_{\mathrm{p} 3}\right)+\left(\frac{D_{2} b_{51}}{D_{1}}-b_{52}\right) h_{\mathrm{p} 1} h_{\mathrm{p} 2}\right) \\
& \frac{1}{h_{\mathrm{p} 3}}\left(\left(\frac{D_{2} b_{41}}{D_{1}}-b_{42}\right) h_{\mathrm{p} 2}+\left(\frac{D_{2} b_{51}}{D_{1}}-b_{52}\right) h_{\mathrm{p} 1}\right), b_{42}-\frac{b_{41} D_{2}}{D_{1}}, b_{52}-\frac{b_{51} D_{2}}{D_{1}}, \\
& \left.\frac{1}{h_{\mathrm{p} 1}}\left(\left(\frac{D_{2} b_{41}}{D_{1}}-b_{42}\right) h_{\mathrm{p} 3}+\left(\frac{D_{2} b_{51}}{D_{1}}-b_{52}\right) h_{\mathrm{p} 2}\right)\right]^{\mathrm{T}}
\end{aligned}
$$




$$
\begin{aligned}
\mathbf{e}_{3}= & {\left[s_{1}-\frac{D_{4} w_{1}}{D_{1}}-\frac{D_{5}\left(t_{1}-\frac{D_{2} w_{1}}{D_{1}}\right)}{D_{3}},\right.} \\
& \frac{1}{h_{\mathrm{p} 3}^{3}}\left(\frac{b_{51}\left(h_{\mathrm{p} 1} h_{\mathrm{p} 2}^{2}-h_{\mathrm{p} 1}^{2} h_{\mathrm{p} 3}\right)-b_{41}\left(h_{\mathrm{p} 2}^{3}-2 h_{\mathrm{p} 1} h_{\mathrm{p} 2} h_{\mathrm{p} 3}\right)}{D_{1}}\left(\frac{D_{5} D_{2}}{D_{3}}-D_{4}\right)+\left(b_{53}-\frac{D_{5}}{D_{3}} b_{52}\right)\left(h_{\mathrm{p} 1} h_{\mathrm{p} 2}^{2}-h_{\mathrm{p} 1}^{2} h_{\mathrm{p} 3}\right)-\right. \\
& \left.-\left(b_{43}-\frac{D_{5}}{D_{3}} b_{42}\right)\left(h_{\mathrm{p} 2}^{3}-2 h_{\mathrm{p} 1} h_{\mathrm{p} 2} h_{\mathrm{p} 3}\right)\right), \frac{1}{h_{\mathrm{p} 3}^{2}}\left(\frac{b_{51} h_{\mathrm{p} 1} h_{\mathrm{p} 2}+b_{41}\left(h_{\mathrm{p} 2}^{2}-h_{\mathrm{p} 1} h_{\mathrm{p} 3}\right)}{D_{1}}\left(\frac{D_{5} D_{2}}{D_{3}}-D_{4}\right)+\right. \\
& \left.+\left(b_{53}-\frac{D_{5}}{D_{3}} b_{52}\right) h_{\mathrm{p} 1} h_{\mathrm{p} 2}+\left(b_{43}-\frac{D_{5}}{D_{3}} b_{42}\right)\left(h_{\mathrm{p} 2}^{2}-h_{\mathrm{p} 1} h_{\mathrm{p} 3}\right)\right), \frac{1}{h_{\mathrm{p} 3}}\left(\frac{b_{51} h_{\mathrm{p} 1}+b_{41} h_{\mathrm{p} 2}}{D_{1}}\left(D_{4}-\frac{D_{5} D_{2}}{D_{3}}\right)-\right. \\
& \left.-\left(b_{53}-\frac{D_{5}}{D_{3}} b_{52}\right) h_{\mathrm{p} 1}-\left(b_{43}-\frac{D_{5}}{D_{3}} b_{42}\right) h_{\mathrm{p} 2}\right), b_{43}-\frac{\left(b_{42}-\frac{b_{41} D_{2}}{D_{1}}\right) D_{5}}{\mathrm{D} 3}-\frac{b_{41} D_{4}}{D_{1}}, \\
& b_{53}-\frac{\left(b_{52}-\frac{b_{51} D_{2}}{D_{1}}\right) D_{5}}{\mathrm{D} 3}-\frac{b_{51} D_{4}}{D_{1}}, \frac{1}{h_{\mathrm{p} 1}}\left(\frac{b_{51} h_{\mathrm{p} 2}+b_{41} h_{\mathrm{p} 3}}{D_{1}}\left(D_{4}-\frac{D_{5} D_{2}}{D_{3}}\right)-\left(b_{53}-\frac{D_{5}}{D_{3}} b_{52}\right) h_{\mathrm{p} 2}-\right. \\
& \left.\left.-\left(b_{43}-\frac{D_{5}}{D_{3}} b_{42}\right) h_{\mathrm{p} 3}\right)\right]^{\mathrm{T}}
\end{aligned}
$$$$
D_{1}=\left|b_{41}\right|^{2}+\left|b_{51}\right|^{2}+|w|^{2}+\left|\frac{b_{51} h_{\mathrm{p} 1}+b_{41} h_{\mathrm{p} 2}}{h_{\mathrm{p} 3}}\right|^{2}+\left|\frac{b_{51} h_{\mathrm{p} 2}+b_{41} h_{\mathrm{p} 3}}{h_{\mathrm{p} 1}}\right|^{2}+\left|\frac{b_{51} h_{\mathrm{p} 1} h_{\mathrm{p} 2}+b_{41}\left(h_{\mathrm{p} 2}^{2}-h_{\mathrm{p} 1} h_{\mathrm{p} 3}\right)}{h_{\mathrm{p} 3}^{2}}\right|^{2}+
$$

$$
\begin{aligned}
& +\left|\frac{b_{51}\left(h_{\mathrm{p} 1} h_{\mathrm{p} 2}^{2}-h_{\mathrm{p} 1}^{2} h_{\mathrm{p} 3}\right)-b_{41}\left(h_{\mathrm{p} 2}^{3}-2 h_{\mathrm{p} 1} h_{\mathrm{p} 2} h_{\mathrm{p} 3}\right)}{h_{\mathrm{p} 3}^{3}}\right|^{2} \\
D_{2}= & b_{42} b_{41}^{*}+b_{52} b_{51}^{*}+t_{1} w_{1}^{2}+\left|\frac{1}{h_{\mathrm{p} 3}}\right|^{2}\left[\left(b_{52} h_{\mathrm{p} 1}+b_{42} h_{\mathrm{p} 2}\right)\left(b_{51} h_{\mathrm{p} 1}+b_{41} h_{\mathrm{p} 2}\right)^{*}\right]+
\end{aligned}
$$$$
+\left|\frac{1}{h_{\mathrm{p} 1}}\right|^{2}\left[\left(b_{52} h_{\mathrm{p} 2}+b_{42} h_{\mathrm{p} 3}\right)\left(b_{51} h_{\mathrm{p} 2}+b_{41} h_{\mathrm{p} 3}\right)^{*}\right]+
$$$$
+\left|\frac{1}{h_{\mathrm{p} 3}}\right|^{4}\left[b_{52} h_{\mathrm{p} 1} h_{\mathrm{p} 2}+b_{42}\left(h_{\mathrm{p} 2}^{2}-h_{\mathrm{p} 1} h_{\mathrm{p} 3}\right)\right]\left[b_{51} h_{\mathrm{p} 1} h_{\mathrm{p} 2}+b_{41}\left(h_{\mathrm{p} 2}^{2}-h_{\mathrm{p} 1} h_{\mathrm{p} 3}\right)\right]^{*}+
$$

$$
\begin{aligned}
& +\left|\frac{1}{h_{\mathrm{p} 3}}\right|^{6}\left[b_{52}\left(h_{\mathrm{p} 1} h_{\mathrm{p} 2}^{2}-h_{\mathrm{p} 1}^{2} h_{\mathrm{p} 3}\right)-b_{42}\left(h_{\mathrm{p} 2}^{3}-2 h_{\mathrm{p} 1} h_{\mathrm{p} 2} h_{\mathrm{p} 3}\right)\right]\left[b_{51}\left(h_{\mathrm{p} 1} h_{\mathrm{p} 2}^{2}-h_{\mathrm{p} 1}^{2} h_{\mathrm{p} 3}\right)-b_{41}\left(h_{\mathrm{p} 2}^{3}-2 h_{\mathrm{p} 1} h_{\mathrm{p} 2} h_{\mathrm{p} 3}\right)\right]^{*} \\
D_{3}= & \left|b_{42}-\frac{b_{41} D_{2}}{D_{1}}\right|^{2}+\left|b_{52}-\frac{b_{51} D_{2}}{D_{1}}\right|^{2}+\left|t_{1}-\frac{D_{2} w_{1}}{D_{1}}\right|^{2}+\left|\frac{1}{h_{\mathrm{p} 3}}\right|^{2}\left|\left(\frac{D_{2} b_{41}}{D_{1}}-b_{42}\right) h_{\mathrm{p} 2}+\left(\frac{D_{2} b_{51}}{D_{1}} b_{52}\right) h_{\mathrm{p} 1}\right|^{2}+ \\
& +\left|\frac{1}{h_{\mathrm{p} 1}}\right|^{2}\left|\left(\frac{D_{2} b_{41}}{D_{1}}-b_{42}\right) h_{\mathrm{p} 3}+\left(\frac{D_{2} b_{51}}{D_{1}}-b_{52}\right) h_{\mathrm{p} 2}\right|^{2}+ \\
& +\left|\frac{1}{h_{\mathrm{p} 3}}\right|^{4}\left|\left(\frac{D_{2} b_{41}}{D_{1}}-b_{42}\right)\left(h_{\mathrm{p} 2}^{2}-h_{\mathrm{p} 1} h_{\mathrm{p} 3}\right)+\left(\frac{D_{2} b_{51}}{D_{1}}-b_{52}\right) h_{\mathrm{p} 1} h_{\mathrm{p} 2}\right|^{2}+ \\
& +\left|\frac{1}{h_{\mathrm{p} 3}}\right|^{6}\left|\left(\frac{D_{2} b_{41}}{D_{1}}-b_{42}\right)\left(h_{\mathrm{p} 2}^{3}-2 h_{\mathrm{p} 1} h_{\mathrm{p} 2} h_{\mathrm{p} 3}\right)-\left(\frac{D_{2} b_{51}}{D_{1}}-b_{52}\right)\left(h_{\mathrm{p} 1} h_{\mathrm{p} 2}^{2}-h_{\mathrm{p} 1}^{2} h_{\mathrm{p} 3}\right)\right|^{2}
\end{aligned}
$$

$D_{4}=b_{43}\left[b_{41}^{*}+b_{53} b_{51}^{*}+s_{1} w_{1}^{*}+\left|\frac{1}{h_{\mathrm{p} 3}}\right|^{2}\left[\left(b_{53} h_{\mathrm{p} 1}+b_{43} h_{\mathrm{p} 2}\right)\left(b_{51} h_{\mathrm{p} 1}+b_{41} h_{\mathrm{p} 2}\right)^{*}\right]+\right.$

$+\left|\frac{1}{h_{\mathrm{p} 1}}\right|^{2}\left[\left(b_{53} h_{\mathrm{p} 2}+b_{43} h_{\mathrm{p} 3}\right)\left(b_{51} h_{\mathrm{p} 2}+b_{41} h_{\mathrm{p} 3}\right)^{*}\right]+$

$+\left|\frac{1}{h_{\mathrm{p} 3}}\right|^{4}\left[b_{53} h_{\mathrm{p} 1} h_{\mathrm{p} 2}+b_{43}\left(h_{\mathrm{p} 2}^{2}-h_{\mathrm{p} 1} h_{\mathrm{p} 3}\right)\right]\left[b_{51} h_{\mathrm{p} 1} h_{\mathrm{p} 2}+b_{41}\left(h_{\mathrm{p} 2}^{2}-h_{\mathrm{p} 1} h_{\mathrm{p} 3}\right)\right]^{*}+$

$+\left|\frac{1}{h_{\mathrm{p} 3}}\right|^{6}\left[b_{53}\left(h_{\mathrm{p} 1} h_{\mathrm{p} 2}^{2}-h_{\mathrm{p} 1}^{2} h_{\mathrm{p} 3}\right)-b_{43}\left(h_{\mathrm{p} 2}^{3}-2 h_{\mathrm{p} 1} h_{\mathrm{p} 2} h_{\mathrm{p} 3}\right)\right]\left[b_{51}\left(h_{\mathrm{p} 1} h_{\mathrm{p} 2}^{2}-h_{\mathrm{p} 1}^{2} h_{\mathrm{p} 3}\right)-b_{41}\left(h_{\mathrm{p} 2}^{3}-2 h_{\mathrm{p} 1} h_{\mathrm{p} 2} h_{\mathrm{p} 3}\right)\right]^{*}$ 


$$
\begin{aligned}
& D_{5}=b_{43}\left(b_{42}^{*}-\frac{D_{2}^{*} b_{41}^{*}}{D_{1}}\right)+b_{53}\left(b_{52}^{*}-\frac{D_{2}^{*} b_{51}^{*}}{D_{1}}\right)+s_{1}\left(t_{1}^{*}-\frac{D_{2}^{*} w_{1}^{*}}{D_{1}}\right)- \\
& -\left|\frac{1}{h_{\mathrm{p} 3}}\right|^{2}\left(b_{53} h_{\mathrm{p} 1}+b_{43} h_{\mathrm{p} 2}\right)\left[\left(\frac{D_{2} b_{51}}{D_{1}}-b_{52}\right) h_{\mathrm{p} 1}+\left(\frac{D_{2} b_{41}}{D_{1}}-b_{42}\right) h_{\mathrm{p} 2}\right]^{*}- \\
& -\left|\frac{1}{h_{\mathrm{p} 1}}\right|^{2}\left(b_{53} h_{\mathrm{p} 2}+b_{43} h_{\mathrm{p} 3}\right)\left[\left(\frac{D_{2} b_{51}}{D_{1}}-b_{52}\right) h_{\mathrm{p} 2}+\left(\frac{D_{2} b_{41}}{D_{1}}-b_{42}\right) h_{\mathrm{p} 3}\right]^{*}- \\
& -\left|\frac{1}{h_{\mathrm{p} 3}}\right|^{4}\left[b_{53} h_{\mathrm{p} 1} h_{\mathrm{p} 2}+b_{43}\left(h_{\mathrm{p} 2}^{2}-h_{\mathrm{p} 1} h_{\mathrm{p} 3}\right)\right]\left[\left(\frac{D_{2} b_{51}}{D_{1}}-b_{52}\right) h_{\mathrm{p} 1} h_{\mathrm{p} 2}+\left(\frac{D_{2} b_{41}}{D_{1}}-b_{42}\right)\left(h_{\mathrm{p} 2}^{2}-h_{\mathrm{p} 1} h_{\mathrm{p} 3}\right)\right]^{*}- \\
& -\left|\frac{1}{h_{\mathrm{p} 3}}\right|^{6}\left[b_{53}\left(h_{\mathrm{p} 1} h_{\mathrm{p} 2}^{2}-h_{\mathrm{p} 1}^{2} h_{\mathrm{p} 3}\right)-b_{43}\left(h_{\mathrm{p} 2}^{3}-2 h_{\mathrm{p} 1} h_{\mathrm{p} 2} h_{\mathrm{p} 3}\right)\right] \\
& {\left[\left(\frac{D_{2} b_{51}}{D_{1}}-b_{52}\right)\left(h_{\mathrm{p} 1} h_{\mathrm{p} 2}^{2}-h_{\mathrm{p} 1}^{2} h_{\mathrm{p} 3}\right)-\left(\frac{D_{2} b_{41}}{D_{1}}-b_{42}\right)\left(h_{\mathrm{p} 2}^{3}-2 h_{\mathrm{p} 1} h_{\mathrm{p} 2} h_{\mathrm{p} 3}\right)\right]^{*}} \\
& D_{6}=\left|b_{43}-\frac{b_{41} D_{4}}{D_{1}}-\frac{\left(b_{42}-\frac{b_{41} D_{2}}{D_{1}}\right) D_{5}}{D_{3}}\right|^{2}+\left|b_{53}-\frac{b_{51} D_{4}}{D_{1}}-\frac{\left(b_{52}-\frac{b_{51} D_{2}}{D_{1}}\right) D_{5}}{D_{3}}\right|^{2}+\left|s_{1}-\frac{D_{4} w_{1}}{D_{1}}-\frac{D_{5}\left(t_{1}-\frac{D_{2} w_{1}}{D_{1}}\right)}{D_{3}}\right|^{2} \\
& +\left|\frac{1}{h_{\mathrm{p} 3}}\right|^{2}\left|\left(D_{4}-\frac{D_{5} D_{2}}{D_{3}}\right) \frac{b_{51} h_{\mathrm{p} 1}+b_{41} h_{\mathrm{p} 2}}{D_{1}}+\left(\frac{D_{5} b_{52}}{D_{3}}-b_{53}\right) h_{\mathrm{p} 1}+\left(\frac{D_{5} b_{42}}{D_{3}}-b_{43}\right) h_{\mathrm{p} 2}\right|^{2}+ \\
& +\left|\frac{1}{h_{\mathrm{p} 1}}\right|^{2}\left|\left(D_{4}-\frac{D_{5} D_{2}}{D_{3}}\right) \frac{b_{51} h_{\mathrm{p} 2}+b_{41} h_{\mathrm{p} 3}}{D_{1}}+\left(\frac{D_{5} b_{52}}{D_{3}}-b_{53}\right) h_{\mathrm{p} 2}+\left(\frac{D_{5} b_{42}}{D_{3}}-b_{43}\right) h_{\mathrm{p} 3}\right|^{2} \\
& +\left|\frac{1}{h_{\mathrm{p} 3}}\right|^{4} \mid\left(\frac{D_{5} D_{2}}{D_{3}}-D_{4}\right) \frac{b_{51} h_{\mathrm{p} 1} h_{\mathrm{p} 2}+b_{41}\left(h_{\mathrm{p} 2}^{2}-h_{\mathrm{p} 1} h_{\mathrm{p} 3}\right)}{D_{1}}-\left(\frac{D_{5} b_{52}}{D_{3}}-b_{53}\right) h_{\mathrm{p} 1} h_{\mathrm{p} 2}- \\
& -\left.\left(\frac{D_{5} b_{42}}{D_{3}}-b_{43}\right)\left(h_{\mathrm{p} 2}^{2}-h_{\mathrm{p} 1} h_{\mathrm{p} 3}\right)\right|^{2}+\left|\frac{1}{h_{\mathrm{p} 3}}\right|^{6} \mid\left(\frac{D_{5} D_{2}}{D_{3}}-D_{4}\right) \frac{b_{51}\left(h_{\mathrm{p} 1} h_{\mathrm{p} 2}^{2}-h_{\mathrm{p} 1}^{2} h_{\mathrm{p} 3}\right)-b_{41}\left(h_{\mathrm{p} 2}^{3}-2 h_{\mathrm{p} 1} h_{\mathrm{p} 2} h_{\mathrm{p} 3}\right)}{D_{1}}- \\
& -\left(\frac{D_{5} b_{52}}{D_{3}}-b_{53}\right)\left(h_{\mathrm{p} 1} h_{\mathrm{p} 2}^{2}-h_{\mathrm{p} 1}^{2} h_{\mathrm{p} 3}\right)+\left.\left(\frac{D_{5} b_{42}}{D_{3}}-b_{43}\right)\left(h_{\mathrm{p} 2}^{3}-2 h_{\mathrm{p} 1} h_{\mathrm{p} 2} h_{\mathrm{p} 3}\right)\right|^{2}
\end{aligned}
$$

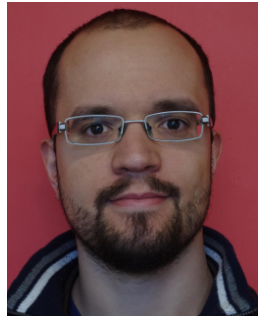

Marco Maso received the bachelor's degree in 2005 and the M.Sc. degree in Telecommunications Engineering in 2008, both from University of Padova, Italy, and is currently pursuing his Ph.D degree at University of Padova and Supélec. He worked on projects dealing with practical implementations of OFDM packet synchronization in 2005/06, and DVB-T2 system simulation in $2008 / 09$. He is currently involved in the HENIAC project, studying new techniques for high speed optical communications. His research interests include heterogeneous networks, wireless communications, cognitive radio and embedded devices.

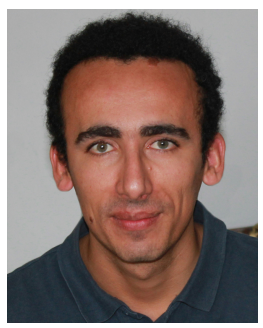

Ejder Bastug received the B.Sc. in Electronic Engineering from Fatih University, Istanbul, Turkey, in 2009. From April 2011 to April 2012, he was an intern researcher at Alcatel-Lucent chair on Flexible radio, Supélec, France. He is currently pursuing his M.Sc. in Electronics Engineering from Fatih University. He is specialized in different aspects of software and electronics engineering. His research interests are in information theory and wireless communications.

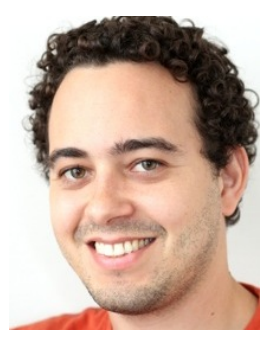

Leonardo S. Cardoso received his electrical engineering and M.Sc. degrees from the Universidade Federal do Ceará (UFC), Brazil in 2003 and 2006, and his Ph.D. degree in 2011 from Supélec, France, on Cognitive Radio and Dynamic Spectrum Access. From 2001 to 2006, he worked in several projects for Ericsson Research at the Wireless Telecommunications Research Group, GTEL, Brazil. In 2006, he joined the Eurécom Institute, France, working in projects on heterogeneous networks and real-time MIMO channel performance assessment, significantly contributing to the EMOS MIMO platform. He is currently a research fellow at INRIA, France, developing a large scale Cognitive Radio testbed.

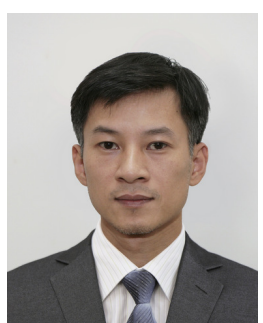

Nguyen Linh-Trung received the B.Eng. and Ph.D. degrees in Electrical Engineering from the Queensland University of Technology, Brisbane, Australia, in 1997 and 2005, respectively. From 2003 to 2005, he was a postdoctoral research fellow at the Centre National d'Etudes Spatiales (CNES), Toulouse, France. Since 2006, he joined the Faculty of Electronics and Telecommunications, University of Engineering and Technology, Vietnam National University, Hanoi, Vietnam. He has held visiting positions at Vanderbilt University, SUPELEC and the University Paris 13. His interest is reduction of data dimension with applications in biomedical engineering and wireless communications, including time-frequency analysis, blind source separation, compressed sensing, network coding. 


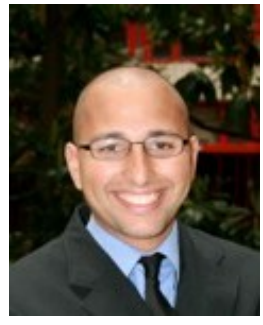

Mérouane Debbah entered the Ecole Normale Supérieure de Cachan (France) in 1996 where he received his M.Sc and Ph.D. degrees respectively. He worked for Motorola Labs (Saclay, France) from 1999-2002 and the Vienna Research Center for Telecommunications (Vienna, Austria) from 2002-2003. He then joined the Mobile Communications department of the Institut Eurecom (Sophia Antipolis, France) as an Assistant Professor. Since 2007, he is a Full Professor at Supelec (Gif-surYvette, France), holder of the Alcatel-Lucent Chair on Flexible Radio. His research interests are in information theory, signal processing and wireless communications. He is an Associate Editor for IEEE Transactions on Signal Processing. Mérouane Debbah is the recipient of the "Mario Boella" prize award in 2005, the 2007 General Symposium IEEE GLOBECOM best paper award, the Wi-Opt 2009 best paper award, the $2010 \mathrm{Newcom++}$ best paper award as well as the Valuetools 2007, Valuetools 2008 and CrownCom2009 best student paper awards. He is a WWRF fellow. In 2011, he received the IEEE/SEE Glavieux Prize Award.

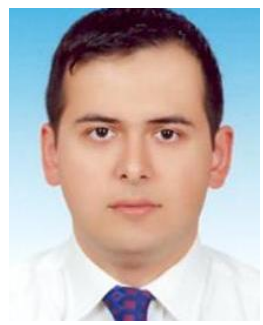

Ozgur Ozdemir received the B.S. degree in Electrical and Electronics Engineering from Bogazici University, Turkey in 1999 and the M.S. and the Ph.D. degrees in Electrical Engineering from The University of Texas at Dallas, Dallas, TX, in 2002 and 2007, respectively. From 2007 to 2011, he worked as an assistant professor in the department of Electrical and Electronics Engineering, Fatih University, Istanbul, Turkey. Since 2011 he is a visiting postdoctoral scholar at Qatar University. His research interests include opportunistic approaches in wireless systems, experimental multiple antenna systems, signal processing, multiuser detection, and digital compensation of RF impairments. 